\title{
Article \\ Radiation Pattern Synthesis of the Coupled Almost Periodic Antenna Arrays Using Artificial Neural Network ANN Model
}

\author{
Hamdi Bilel *(1) and Aguili Taoufik \\ Communication System Laboratory Sys'Com, National Engineering School of Tunis (ENIT), University of Tunis \\ El Manar , BP 37, Le Belvédère, Tunis 1002, Tunisia; taoufik.aguili@gmail.com \\ * Correspondence: hbilel.enit@gmail.com; Tel.: +32-466422407
}

check for

updates

Citation: Bilel, H.; Taoufik, A.

Radiation Pattern Synthesis of the Coupled Almost Periodic Antenna Arrays Using Artificial Neural Network ANN Model. Preprints 2022, 1, 0. https://doi.org/

Publisher's Note: MDPI stays neutral with regard to jurisdictional claims in published maps and institutional affiliations.

Copyright: (C) 2022 by the authors Licensee MDPI, Basel, Switzerland. This article is an open access article distributed under the terms and conditions of the Creative Commons Attribution (CC BY) license (https:// creativecommons.org/licenses/by/ $4.0 /)$.

\begin{abstract}
This paper proposes a radiation pattern synthesis of the almost periodic antenna arrays including mutual coupling effects (that extracted by the Floquet analysis according to our previous work), which principally has a high directivity and large bandwidth. For modeling the given structures, the moment method combined with the Generalized Equivalent Circuit (MoM-GEC) is proposed. The artificial neural network (ANN) as a powerful computational model has been successfully applied to the antenna array pattern synthesis. The results showed that the multilayer feedforward neural networks are rugged and can successfully and efficiently resolve various distinctive complex almost periodic antenna patterns (with different source amplitudes) (in particular, both periodic and randomly aperiodic structures are taken into account). However, the artificial neural network (ANN) is capable of quickly producing the synthesis results using generalization with the early stopping (ES) method. A significant time gain and memory consumption are achieved by using this given method to improve the generalization (called early stopping). To justify this work, several examples are developed and discussed.
\end{abstract}

Keywords: Radiation pattern synthesis;Almost periodic structures; Mutual coupling effects; Artificial neural network ANN algorithm; Early stoping method.

\section{Introduction}

Today, the synthesis of the radiation pattern of almost periodic planar structures is the subject of various scientific researches, mostly in space and defense applications, communication systems and electronic devices such as phased array radar systems, frequency selective surface (FSS) applications [28], millimeter waves, and optical wave regions (for other examples: reflection gratings, phased arrays, and electromagnetic bandgap structures, leaky wave antennas, etc.) $[9,11,14]$.

In general, smart antenna arrays involve intelligent systems including genetic algorithms and neural networks to synthesize the radiation pattern [2,3]. Many research papers are proving that genetic algorithm (GA) is used basically for sidelobe reduction in antenna pattern synthesis $[15,18]$. As well, artificial neural networks (ANN) have been employed in various purposes such as pattern recognition systems, and have been taken into use for input-output mapping, system identification, adaptive prediction, etc..... Consequently, the present work is centered on the neural network technique that will be implemented for the synthesis of almost periodic network models, pointing out their most prominent features and distinctive characteristics $[17,24,25]$.

The objective is then to develop a feedforward neural network with supervised training approximating the function of the radiation pattern which is provided by the method of moments simplified by the equivalent circuit scheme.

For clarity, this paper presents some basic details on the optimum size of a feedforward neural network to prevent overfitting problems. Next, the radiation model datasets will be divided for training, testing, and validation, and then a feedforward neural network will be generated and trained [21]. The output values will be built and denormalized, and finally, the neural network performance will be examined by checking the output values against the numerical target values [24,25].

This fact reduces the complexness of the problem by setting and adapting the neural network patterns, such as training function, architecture, and parameter, which would enhance 
and give more accuracy about the input-output relations [18]. Our main idea here is to address the synthesis of antenna arrays for coupled almost periodic array geometries and especially in future complex extended aperiodic configurations [19,32].

This paper is organized as follows: in the section 2, the fundamental theoretical background of numerical analysis including the computation of the radiation field is reminded, particularly for almost periodic arrays. To synthesize the radiation pattern, the basic concepts of artificial neural networks (ANN) and their applications are recalled in the section 3. It describes how to introduce the basic principles of artificial neural networks (ANNs). Some fundamental networks are examined in detail for their capability to deal with the problem of simple pattern synthesis. These fundamental networks, together with the principles of ANNs, will lead to the development and construction of new architectures for the synthesis of complex almost periodic antenna arrays (e.g. randomly aperiodic phased arrays). The next section illustrates the numerical results and discussions. In the last section, some conclusions are presented.

\section{Problem Formulation: (Radiation pattern of the almost periodic structures)}

This section takes up the main expression of the radiation pattern related to the proposed almost periodic planar structures (see figure (1) of the periodic antenna array example) $[6-8]$ which is discussed in our previous work $[5,9,11]$. To resolve this problem, it is claimed that an integral method based on the method of moments combined with the generalized equivalent circuit (MoM-GEC) $[6,7,10]$ is implemented.

Therefore, the relation of the total near-field distribution set on the surface of the discontinuity (rectangular opening aperture) (metal form + dielectric substrate) of the global almost periodic structures can be defined according to the Galerkin procedure as follows [6-8,14]:

- On the metallic part, the electric field can be given by:

$$
|E(x, y)\rangle=\sum_{p} \sum_{q}[Z]_{p q} X_{p q}\left|g_{p q}\right\rangle
$$

- On the discontinuity plane (or on the radiating aperture), the electric field is expressed in function of the guide's modes as :

$$
|E(x, y)\rangle=\sum_{m, n} \sum_{p} \sum_{q}[Z]_{p q} X_{p q}\left\langle f_{m n} \mid g_{p q}\right\rangle\left|f_{m n}\right\rangle
$$

with:

$$
\left[\hat{Z}_{p q, s t}^{u p p e r, \text { down }}\right]=\left[\sum_{m, n}\left\langle g_{p q} \mid f_{m n}\right\rangle z_{m n}^{u p p e r, d o w n}\left\langle f_{m n} \mid g_{s t}\right\rangle\right]
$$

and the Inner product is yielded by :

$$
\langle u \mid v\rangle=\iint_{D} u v^{*} d s
$$

( ${ }^{*}$ refers to the complex conjugate)

$\left|f_{m n}\right\rangle$ denotes the modes $\left|T E_{m n}\right\rangle,\left|T M_{m n}\right\rangle$.

$\left|g_{p q}\right\rangle$ indicates the test functions used and $X_{p q}$ the inconnue weights of this function to be evaluated and $\tilde{z}_{m n}$ are the total modal impedance related to the guide modes.

A specific choice of test functions that express the unknown current density established on the metal parts of the selected structures.

Based on the Fourier Transform, the far field of the given total structure can be written as:

$$
\begin{array}{r}
\tilde{E}_{x, y, \theta_{0}, \phi_{0}}(\theta, \phi)=\int_{-c}^{c}\left\{\int_{0}^{L} E_{\text {aperture }}(x, y)\right. \\
\left.e^{\left(j k_{0}\left(\sin (\theta) \cos (\phi)-\sin \left(\theta_{0}\right) \cos \left(\phi_{0}\right)\right) x\right)} d x\right\} \\
e^{\left(j k_{0}\left(\sin (\theta) \sin (\phi)-\sin \left(\theta_{0}\right) \sin \left(\phi_{0}\right)\right) y\right)} d y
\end{array}
$$




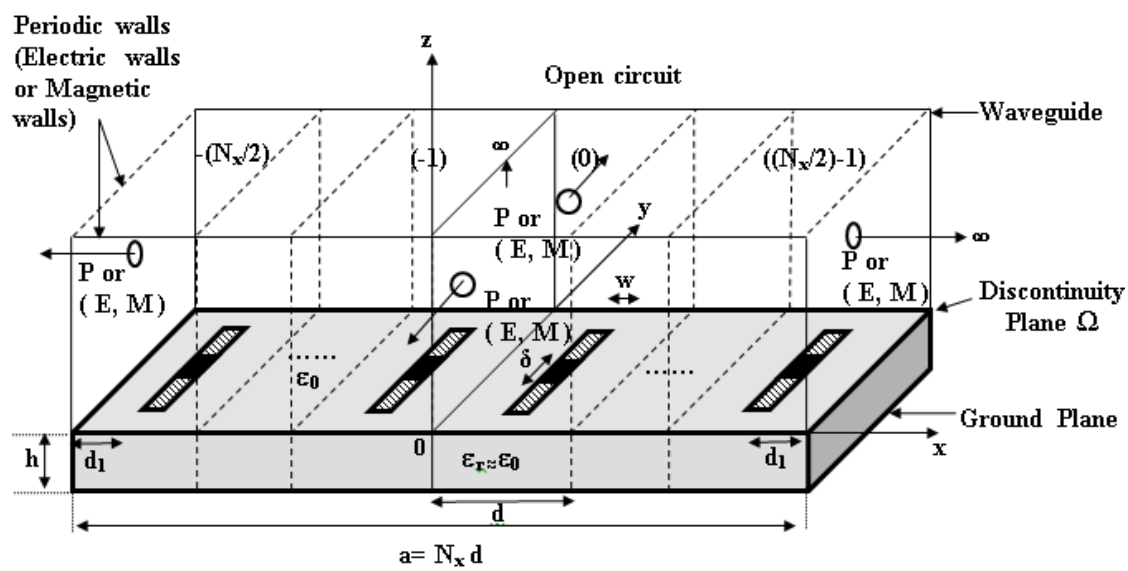

Figure 1. Section of periodic planar phased array dipoles: the walls of the environment can be selected from EEEE ( waveguide with electric walls), EMEM (waveguide with two electric walls and two magnetic walls), EPEP ( waveguide with two electric walls and two periodic walls), PPPP ( waveguide with periodic walls)

$E_{\text {aperture }}(x, y)$ is obtained by a spatial calculation of the MoM method. The pair $\left(\theta_{0}, \phi_{0}\right)$ is seen as the orientation direction of the main beam (steering direction) and the pair $(\theta, \phi)$ indicates the shape of the radiation pattern.

To restraint the electromagnetic calculation on one unit cell, the dependence on Floquet modes $(\alpha$ or $(\alpha, \beta))$ is taken into account $[9,11,14,32]$. Then, the field components can be therefore expressed in the generalized Fourier series expansions, and the analysis region can be reduced to only one periodicity cell bounded by the known periodic walls, as presented in the figure (2). In this case, the interaction between cells can be taken into consideration using a novel expression of the mutual coupling shown in the previous work $[9,11]$. All details about the electromagnetic modal calculation based on the Floquet analysis are proven in $[9,11,14]$. To remind the radiating pattern expression $\tilde{E}_{x, y}(\theta, \phi)$ developed through the modal decomposition in the spectral domain, we can detail the following steps given below [14]:

The total electric field radiated by the structure is:

$$
E(x, y)_{\text {aperture }}=\frac{1}{\sqrt{N_{x} N_{y}}} \sum_{p=-\frac{N_{x}}{2}, q=-\frac{N_{y}}{2}}^{\frac{N_{x}}{2}-1, \frac{N_{y}}{2}-1} \tilde{E}_{\alpha_{p}, \beta_{q}, \text { aperture }}(x, y) e^{j \alpha_{p}\left(i d_{x}\right)} e^{j \beta_{q}\left(s d_{y}\right)}
$$

Then, the normalised radiating pattern is given by:

$$
\begin{array}{r}
\tilde{E}_{x, y, \theta_{0}, \phi_{0}}(\theta, \phi)==\frac{1}{\sqrt{N_{x} N_{y}}} \sum_{i=-\frac{N_{x}}{2}, s=-\frac{N_{y}}{2}}^{\frac{N_{x}}{2}-1, \frac{N_{y}}{2}-1} \\
E_{\text {aperture }}(x, y) e^{\left(j k_{0}\left(\sin (\theta) \cos (\phi)-\sin \left(\theta_{0}\right) \cos \left(\phi_{0}\right)\right) i d_{x}\right)} \\
e^{\left(j k_{0}\left(\sin (\theta) \sin (\phi)-\sin \left(\theta_{0}\right) \sin \left(\phi_{0}\right)\right) \sin y\right)}
\end{array}
$$




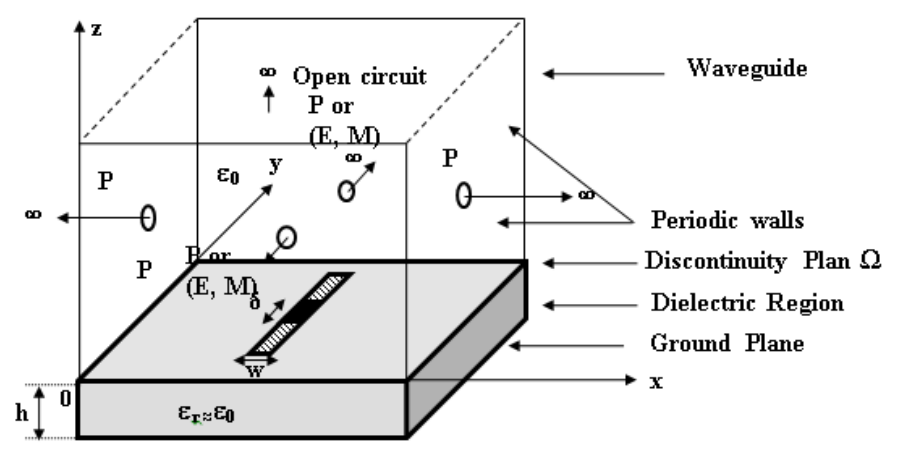

Figure 2. Section of periodic phased array microstrip lines (planar dipoles):(unit cell delimited by periodic walls)

Moreover, the far radiating field made in the Floquet domain when the $z>0$ region, is written as follows:

$$
\begin{gathered}
\tilde{E}_{x, y}^{\alpha, \beta}(\theta, \phi)=\int_{-c}^{c}\left\{\int_{0}^{L} E_{\text {aperture }}^{\alpha, \beta}(x, y)\right. \\
\left.e^{\left(j k_{0}\left(\sin (\theta) \cos (\phi)-\sin \left(\theta_{0}\right) \cos \left(\phi_{0}\right)\right) x\right)} d x\right\} \\
e^{\left(j k_{0}\left(\sin (\theta) \sin (\phi)-\sin \left(\theta_{0}\right) \sin \left(\phi_{0}\right)\right) y\right)} d y
\end{gathered}
$$

After that, the expression of the directivity (in Floquet space) is obtained as [31]:

$$
D_{\alpha, \beta}\left(\phi_{0}, \theta_{0}\right)=\frac{\left|\tilde{E}_{\alpha, \beta}\left(\phi_{0}, \theta_{0}\right)\right|^{2}}{\frac{1}{4 \pi} \int_{0}^{2 \pi} \int_{0}^{\pi}\left|\tilde{E}_{\alpha, \beta}(\theta, \phi)\right|^{2} \sin (\theta) d \phi d \theta}
$$

Therefore, to study the spatial electromagnetic behavior of periodic antenna arrays (as explained in the previous section in Equation (6)), another way to proceed based on the superposition of Floquet states (superposition theorem) in a finite or infinite periodic array

- For small and large finite arrays:

We must indicate that the Floquet states are considered discrete (with their physical fields): The spatial electric and current fields are deduced as:

$$
\begin{array}{r}
E\left(i d_{x}, k d_{y}\right)=\frac{1}{\sqrt{N_{x} N_{y}}} \sum_{p=-\frac{N_{x}}{2}}^{\frac{N_{x}}{2}-1} \sum_{q=-\frac{N_{y}}{2}}^{\frac{N_{y}}{2}-1} \tilde{E}_{\text {aperture }}^{\alpha_{p}, \beta_{q}}(x, y) \\
e^{j \alpha_{p}\left(i d_{x}\right)} e^{j \beta_{q}\left(k d_{y}\right)} \\
J\left(i d_{x}, k d_{y}\right)=\frac{1}{\sqrt{N_{x} N_{y}}} \sum_{p=-\frac{N_{x}}{2}}^{\frac{N_{x}}{2}-1} \sum_{q=-\frac{N_{y}}{2}}^{\frac{N_{y}}{2}-1} \tilde{J}_{\text {aperture }}^{\alpha_{p}, \beta_{q}}(x, y) \\
e^{j \alpha_{p}\left(i d_{x}\right)} e^{j \beta_{q}\left(k d_{y}\right)}
\end{array}
$$

Then, the total radiating electric field is:

$$
E(\theta, \phi)=\frac{1}{\sqrt{N_{x} N_{y}}} \sum_{p=-\frac{N_{x}}{2}}^{\frac{N_{x}}{2}-1} \sum_{q=-\frac{N_{y}}{2}}^{\frac{N_{y}}{2}-1} \tilde{E}_{\alpha_{p}, \beta_{q}}^{x, y, \theta_{0}, \phi_{0}}(\theta, \phi)
$$


- For infinite arrays:

The Floquet states are taken as continuous values which belong to brouillin domain $\alpha \in$ $\left[-\frac{\pi}{d_{x}}, \frac{\pi}{d_{x}}\right], \beta \in\left[-\frac{\pi}{d_{y}}, \frac{\pi}{d_{y}}\right]$. Then, the spatial electric and current fields are defined as:

$$
\begin{gathered}
J(x, y)=\frac{d_{x} d_{y}}{4 \pi^{2}} \int_{\frac{-\pi}{d x}}^{\frac{\pi}{d x}} \int_{\frac{-\pi}{d_{y}}}^{\frac{\pi}{d y}} \tilde{J}_{\text {aperture }}^{\alpha, \beta}(x, y) e^{j \alpha x} e^{j \beta y} d \alpha d \beta \\
E(x, y)=\frac{d_{x} d_{y}}{4 \pi^{2}} \int_{\frac{-\pi}{d_{x}}}^{\frac{\pi}{d x}} \int_{\frac{-\pi}{d_{y}}}^{\frac{\pi}{d_{y}}} \tilde{E}_{\text {aperture }}^{\alpha, \beta}(x, y) e^{j \alpha x} e^{j \beta y} d \alpha d \beta
\end{gathered}
$$

Consequently, the radiation field is written as:

$$
E(\theta, \phi)=\frac{d_{x} d_{y}}{4 \pi^{2}} \int_{\frac{-\pi}{d_{x}}}^{\frac{\pi}{d x}} \int_{\frac{-\pi}{d_{y}}}^{\frac{\pi}{d y}} \tilde{E}_{\alpha, \beta}^{x, y, \theta_{0}, \phi_{0}}(\theta, \phi) d \alpha d \beta
$$

The directivity values can always obey the superposition theorem in the finite and infinite cases, as has been proven in the electric, current, and radiation fields.

A well-detailed explanation of Floquet's modal analysis and its results has been proven in [31].

To study the aperiodic configuration, it is possible to change the amplitudes $\backslash$ phases weights between periodic elements. Note that the electromagnetic calculation remains valid with some modification at the level of the excitation values.

So, the numerical electromagnetic radiation pattern of the whole coupled almost periodic array can be presented as database content to build a feed-forward neural network training with supervised learning that approximates the following array pattern's function $[13,16$, 17].

\section{Artificial neural networks (ANN) principle}

As we have previously seen in our previous work, this section explains how to conjoin the application of neural networks and almost periodic antenna array systems $[13,16,17]$. A neural network is a way to model any input to the output by establishing connections between their related radiation model data, especially if nothing is known about the model. By using the MATLAB tool, this neural processing can show a simple explanation of the model. In fact, the neural network technique is commonly used in two stages: the training phase and the performance phase. For more precision, the MLP network architecture structure is fixed: the number of hidden layers and neurons (nodes) in each layer. The activation functions of each layer are also chosen at this stage, i.e. they are supposed to be known. The unknown parameters to be estimated are the weights and biases. Many algorithms exist to define the network parameters. Here, we will focus only on the use of Levenberg-Marquardt which is generally more efficient but requires more computer memory [1],[22]-[27]. We briefly summarize the learning algorithm procedure for multilayer perceptron networks:

1. The structure of the network is first defined. In the network, activation functions are chosen and the network parameters, weights, and biases are initialized.

2. The parameters associated with the training algorithm like error goal, the maximum number of epochs (iterations), etc, are defined.

3. The training algorithm is called.

4. Once the neural network is determined, the result is first tested by simulating the output of the neural network with the measured input data. This result is compared to the measured output. The final validation must be performed with independent data.

In general, three different types of data sets (inputs) are employed, known as the training set, validation set, and test set. The training set is a set of values that hold information about the target function for training the network. The validation set is assigned to the 
early stopping technique. During the training phase, the validation error is monitored to prevent the network from overfitting the training data. Normally, the test set is just used to assess the performance of the network afterward. $60 \%$ of the data is used for training the network and the remaining $40 \%$ is used equally for validating and testing the network on unlearned inputs respectively $[13,18]$. Specifically, the application randomly divides the input vectors and target vectors into three known sets as follows: The input vectors and target vectors are divided into three known sets.

- $60 \%$ are used for training.

- $20 \%$ are used to validate that the network is generalizing and to stop training before overfitting.

- The last $20 \%$ is used as a completely independent test of network generalization.

Thus, the structure of the network was provided in figures (3)and (4), when the learning phase allowed to elaborate the synaptic weights taken from each formal neuron. In this example, we are using a feed-forward network with a default tan-sigmoid transfer function in the hidden layer and a linear transfer function in the output layer.

The table (1) lists the training parameters of the artificial neural network (ANN) and the

Table 1. Artificial neural network (ANN) training parameters

\begin{tabular}{lc}
\hline Number of input neurons & 100 \\
Number of hidden layers & 2 \\
Number of output neurons & 1 \\
Algorithm & $1 \mathrm{~m}$ \\
Learning rate & 0.01 \\
Momentum & 0.95 \\
MSE goal & $1 e^{-3}$ \\
Minimum performance gradient & $1 e^{-5}$ \\
Initial mu & 0.001 \\
mu decrease factor & 0.1 \\
mu increase factor & 10 \\
Maximum mu & $1 e^{10}$ \\
Epochs between displays & 25 \\
Generate command-line output & false \\
Show training GUI & true \\
Maximum time to train in seconds & inf \\
Maximum number of epochs & 300 \\
Regularization parameter & 0.8 \\
Transfer function in hidden layer & tan-sigmoid ("tansig") \\
Transfer function in output layer & linear("purelin") \\
\hline
\end{tabular}

architectural parameters prepared for the implementation of the artificial neural network (ANN) models.[13,18].

After the training phase, the performance phases of the almost periodic array are accomplished to obtain the optimal weights for the new incoming signals. The performance analysis of this application can be examined by changing the number of antenna elements, the radiation value, the target applications and the angular separation between the incoming signals[24,25].

In this work, the Levenberg Marquardt minimization algorithm (LMA) has been checked to train the artificial neural network (ANN). The accuracy of its models is evaluated by the mean sum of squared error (MSE) between the calculated (desired) radiation pattern obtained using numerical analysis and the predicted values for the training data set [24]. To perform some analysis of the network response, we can perform a linear regression between the network outputs and the corresponding targets [26]. 


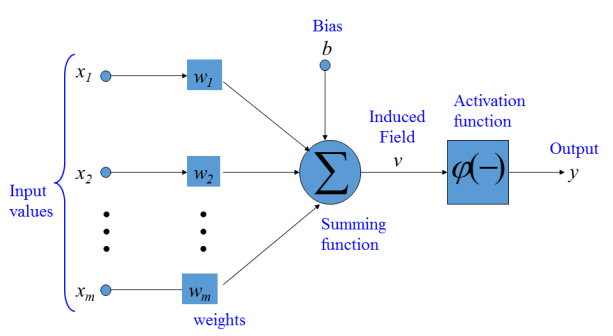

Figure 3. Basic structure of an artificial neuron

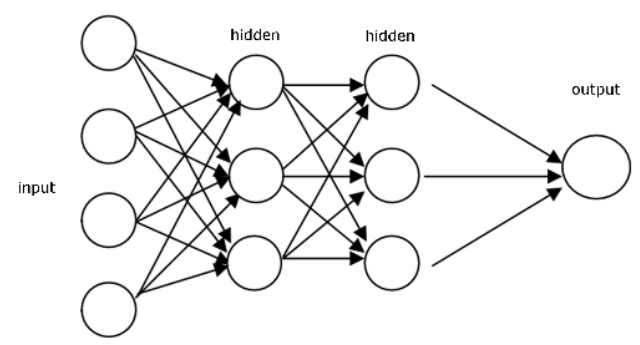

Figure 4. An illustration of a typical feedforward network

\section{Results and observation}

\subsection{Numerical results}

After studying the convergence level of the input impedance and the current distributions in function of the guide's modes and the trial functions, as explained in the past work to demonstrate the coupling terms of the almost periodic array $[9,11]$. This paper focused essentially to create the radiation pattern associated with the planar almost periodic structures [6-8]. At first, the electromagnetic states in electric and current fields of the proposed configuration examples are drawn, that presented at the waveguide aperture. Then, the desired radiation pattern (or the electric far-field) can be computed using the main Fourier expression written and explained in the previous section [6-8,14], where the near field distribution that defined on the discontinuity plane is weighted by a numerical MoM-GEC formulation [5,9,11].
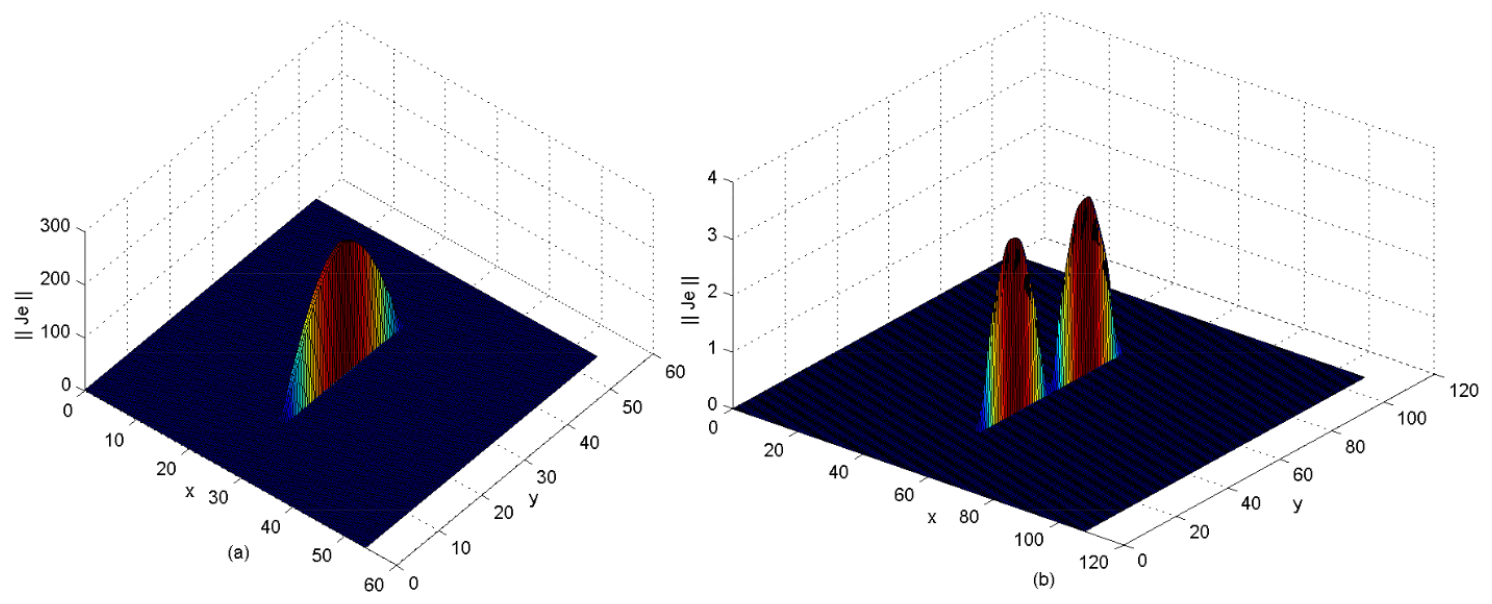

Figure 5. Current density for a unit cell of an almost periodic 2D array (half-wave and full-wave dipoles) defined over the guide aperture, described by the test functions using : $\mathrm{f}=5.4 \mathrm{Ghz}, \alpha_{0}=0 \mathrm{rad}$ $\left.m^{-1}, \beta_{0}=0 \mathrm{rad} m^{-1}, w=1 \mathrm{~mm}, \delta=0.75 \mathrm{~mm}\left(w \ll \lambda_{0}, \delta \ll \lambda_{0}\right), d_{x}=108 \mathrm{~mm}, d_{y}=108 \mathrm{~mm}, a\right) L=$ $\left.\frac{\lambda_{0}}{2} \approx 27 \mathrm{~mm}-b\right) L=\lambda_{0} \approx 54 \mathrm{~mm}, h=1.25 \mathrm{~mm}$ and $\epsilon=\epsilon_{r}=1$ (air)(see to [28] as a comparison reference).

The figures (5),(6)and(7) illustrate numerical current densities and their corresponding electric fields that are calculated for a unit cell of a periodic planar dipole antenna that surrounded by periodic walls. As a result, the far-field of the given unit structure can be described by the following figure(8)[14,20]. [5,9,11].

In this way, the preceding case of the basic element would be generalized to almost periodic array configuration $[6-9,11]$. Thus, based on the formulation problem detailed in $[9,11]$, the electromagnetic fields (E, J) of the whole structure would be shown. So, the figures (9),(10) and (11) allow to verify all the proposed boundaries conditions in term current and electric fields associated to periodic planar structure. 


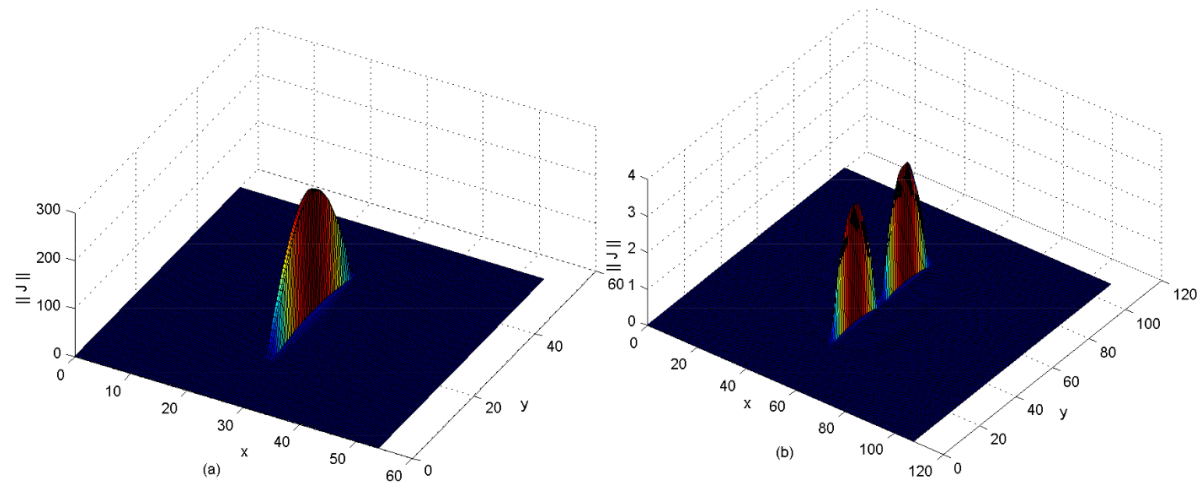

Figure 6. Current density for a unit cell of $2 \mathrm{D}$ almost periodic array (half and full wave dipoles) defined on the guide wave's aperture, described by the basis functions (guide's modes) using: $\mathrm{f}=5.4 \mathrm{Ghz}, \alpha_{0}=0 \mathrm{rad} m^{-1}, \beta_{0}=0 \mathrm{rad} m^{-1}, w=1 \mathrm{~mm}, \delta=0.75 \mathrm{~mm}\left(w \ll \lambda_{0}, \delta \ll \lambda_{0}\right), d_{x}=$ $\left.\left.108 \mathrm{~mm}, d_{y}=108 \mathrm{~mm}, a\right) L=\frac{\lambda_{0}}{2} \approx 27 \mathrm{~mm}-b\right) L=\lambda_{0} \approx 54 \mathrm{~mm}, h=1.25 \mathrm{~mm}$ and $\epsilon=\epsilon_{r}=1$ (air) (see to [28] as a comparison reference).
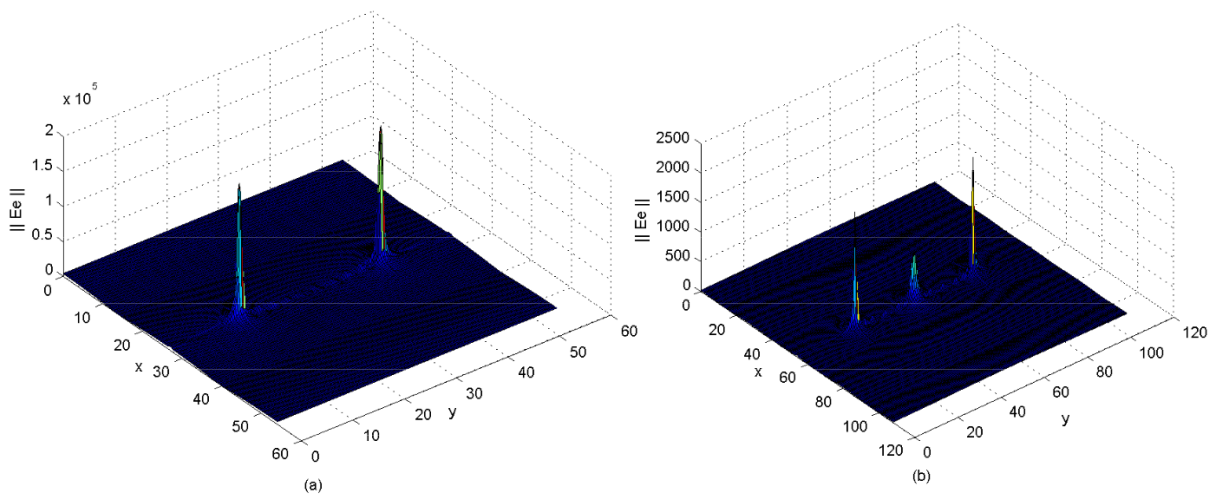

Figure 7. Electric field of 2D almost periodic array's unit cell (half and full wave dipoles) defined on the guide wave's aperture, described by the basis functions (guide's modes) using: $\mathrm{f}=5.4 \mathrm{Ghz}$, $\alpha_{0}=0 \mathrm{rad} m^{-1}, \beta_{0}=0 \mathrm{rad} m^{-1}, w=1 \mathrm{~mm}, \delta=0.75 \mathrm{~mm}\left(w \ll \lambda_{0}, \delta \ll \lambda_{0}\right), d_{x}=108 \mathrm{~mm}, d_{y}=$ $\left.108 \mathrm{~mm}, a) L=\frac{\lambda_{0}}{2} \approx 27 \mathrm{~mm}-b\right) L=\lambda_{0} \approx 54 \mathrm{~mm}, h=1.25 \mathrm{~mm}$ and $\epsilon=\epsilon_{r}=1$ (air) (see to [28] as a comparison reference).

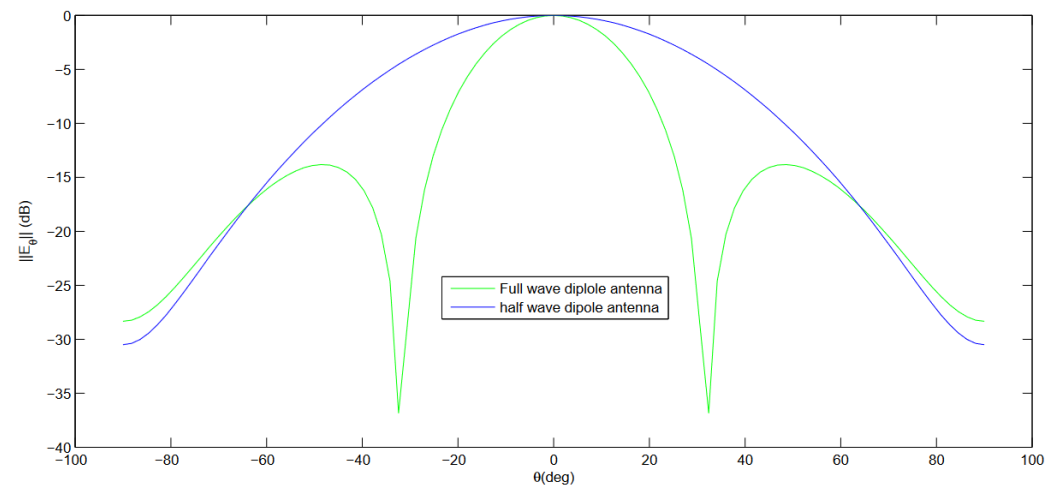

Figure 8. Radiation pattern calculated using the MoM-GEC method for a half-wave and full-wave dipole antenna: $\mathrm{f}=5.4 \mathrm{Ghz}, \alpha_{0}=0 \mathrm{rad} m^{-1}, \beta_{0}=0 \operatorname{rad~} m^{-1}, w=1 \mathrm{~mm}, \delta=0.75 \mathrm{~mm}\left(w \ll \lambda_{0}, \delta \ll\right.$ $\left.\left.\left.\lambda_{0}\right), d_{x}=108 \mathrm{~mm}, d_{y}=108 \mathrm{~mm}, a\right) L=\frac{\lambda_{0}}{2} \approx 27 \mathrm{~mm}-b\right) L=\lambda_{0} \approx 54 \mathrm{~mm}, h=1.25 \mathrm{~mm}$ and $\epsilon=\epsilon_{r}=1$ (air). 
Identically, the $1 \mathrm{D}$ and $2 \mathrm{D}$ quasi-periodic structures follow the same manner to represent the current and electric fields, as indicated in the figures (12), (13), (14), (15) and (16). It's observed that the electromagnetic coupling is expressed by the lying of weak current surface on the non-exciting elements of the aperiodic configuration (which explains also the phenomenon of leaky waves). As shown in the figure(13), a Gibbs effect appears when the guide's modes number is not correctly reached the convergence level.
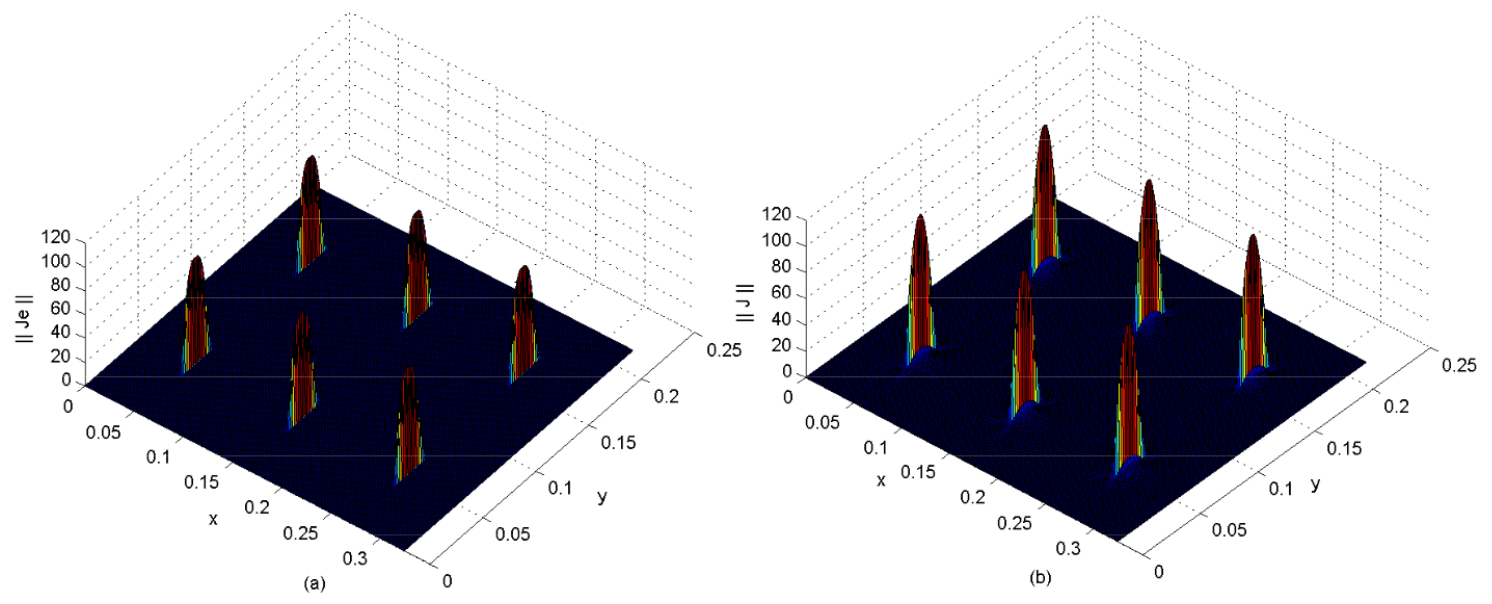

Figure 9. Current density of an example of almost periodic 2D array (half-wave dipoles) defined on the guide wave aperture, described by a)test functions b)basis functions (guide modes) at the operating frequency $\mathrm{f}=5.4 \mathrm{GHz}$. The physical parameters used are : $\alpha_{0}=0 \mathrm{rad} m^{-1}, \beta_{0}=0 \mathrm{rad} m^{-1}$, $w=1 \mathrm{~mm}, \delta=0.75 \mathrm{~mm}\left(w \ll \lambda_{0}, \delta \ll \lambda_{0}\right), d_{x}=108 \mathrm{~mm}, d_{y}=108 \mathrm{~mm}, L=\frac{\lambda_{0}}{2} \approx 27 \mathrm{~mm}, h=1.25 \mathrm{~mm}$ and $\epsilon=\epsilon_{r}=1$ (air) (see to [28] as a comparison reference).
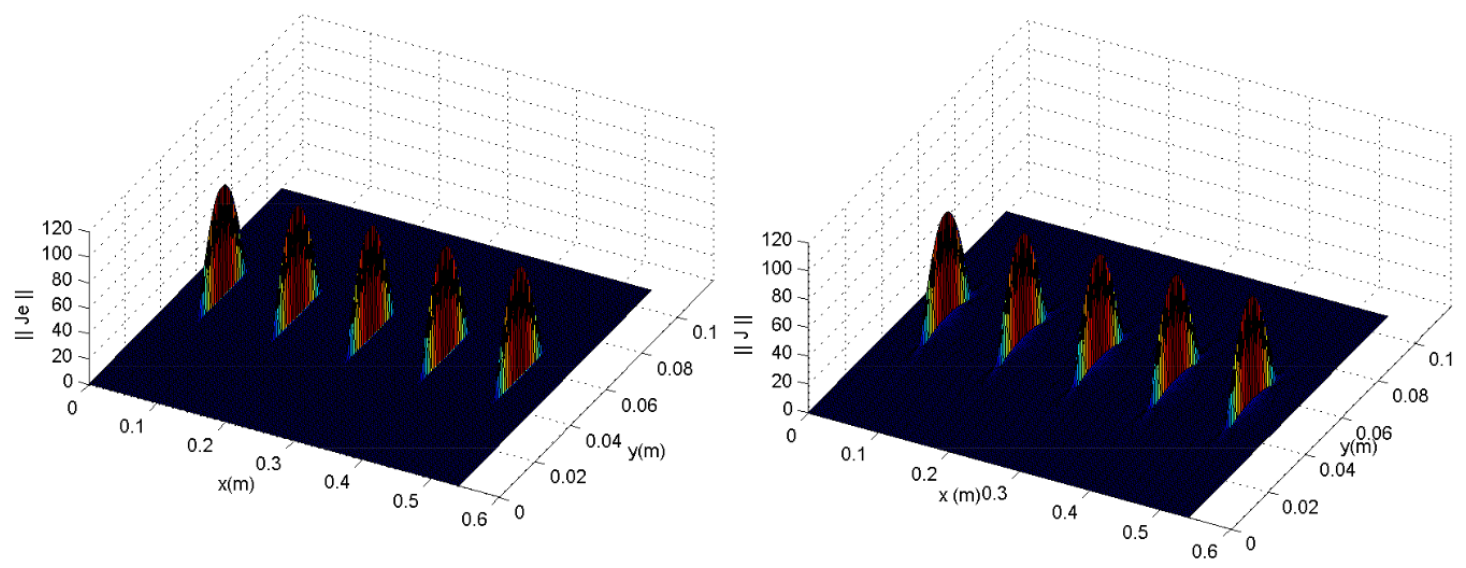

Figure 10. Current density of the almost periodic network (half-wave dipoles) defined on the aperture of the waveguide, described by a)the test functions b)the basic functions (guide modes) at the operating frequency $\mathrm{f}=5.4 \mathrm{GHz}$. The physical parameters used are $: \alpha_{0}=0 \mathrm{rad} \mathrm{m}-1, \beta_{0}=0 \mathrm{rad} \mathrm{m}^{-1}$, $w=1 \mathrm{~mm}, \delta=0.75 \mathrm{~mm}\left(w \ll \lambda_{0}, \delta \ll \lambda_{0}\right), d_{x}=108 \mathrm{~mm}, d_{y}=108 \mathrm{~mm}, L=\frac{\lambda_{0}}{2} \approx 27 \mathrm{~mm}, h=1.25 \mathrm{~mm}$ and $\epsilon=\epsilon_{r}=1$ (air) (see to [28] as a comparison reference).

In the same way, the radiating pattern of the whole planar almost periodic dipole antenna array can be given by the figures (17),(18) and (19). The mutual coupling effects are taken into account by varying the separation periods and the number of elements when the electromagnetic calculation is achieved, as illustrated by the same figures.

According to the figures (17),(18), the proposed structure offers high directivity with a reduced main radiation beam, but suffers from a high sidelobe level (SLL), especially, when the number of elements is grown up and the array elements are decoupled (has a separation period $\left(d_{x} \gg \lambda\right)$. The good radiation pattern description is observed in both E-plane and 
$\mathrm{H}$-plane cuts at the operative frequency $\mathrm{f}=5.4 \mathrm{GHz}$ as shown in figure(19).

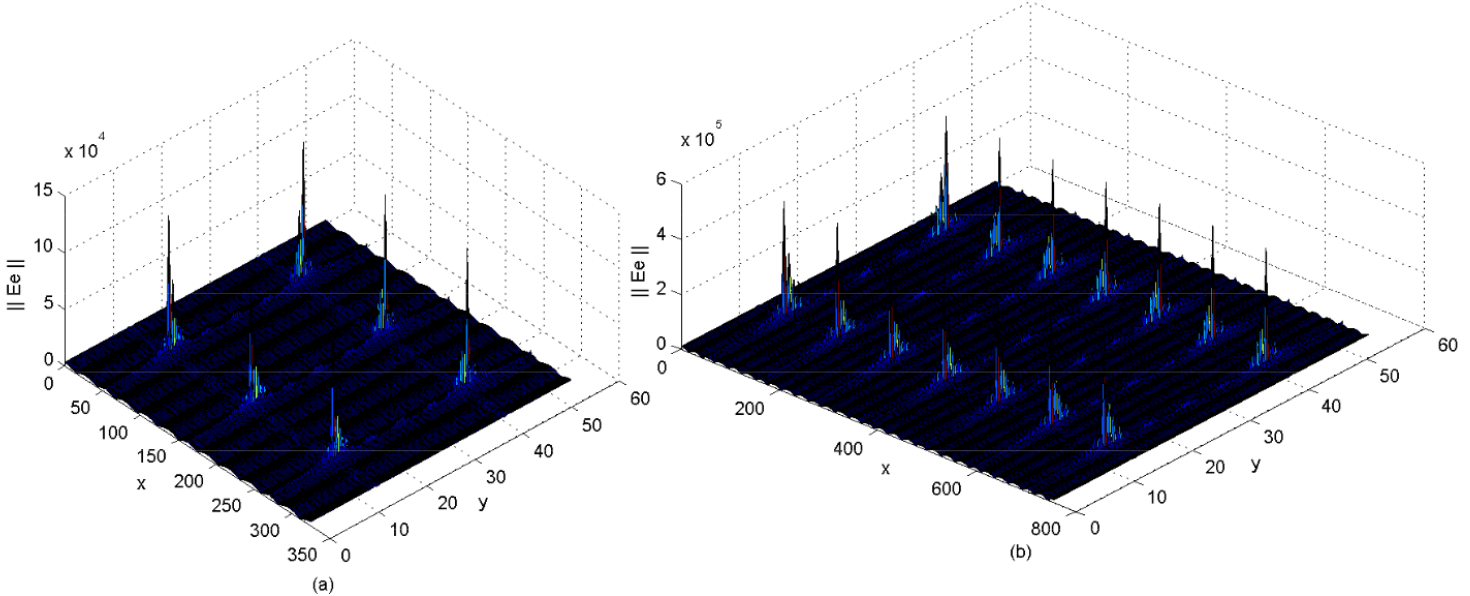

Figure 11. Electric field of the almost periodic array (half wave dipoles)defined on the waveguide's aperture, described by the basis functions (guide's modes) using a)three cells b)seven cells at the operating frequency $\mathrm{f}=5.4 \mathrm{GHz}$. The chosen physical parameters are: $\alpha_{0}=0 \mathrm{rad} m^{-1}, \beta_{0}=0 \mathrm{rad} m^{-1}$, $w=1 \mathrm{~mm}, \delta=0.75 \mathrm{~mm}\left(w \ll \lambda_{0}, \delta \ll \lambda_{0}\right), d_{x}=108 \mathrm{~mm}, d_{y}=108 \mathrm{~mm}, L=\frac{\lambda_{0}}{2} \approx 27 \mathrm{~mm}, h=1.25 \mathrm{~mm}$ and $\epsilon=\epsilon_{r}=1$ (air) (see to [28] as a comparison reference).

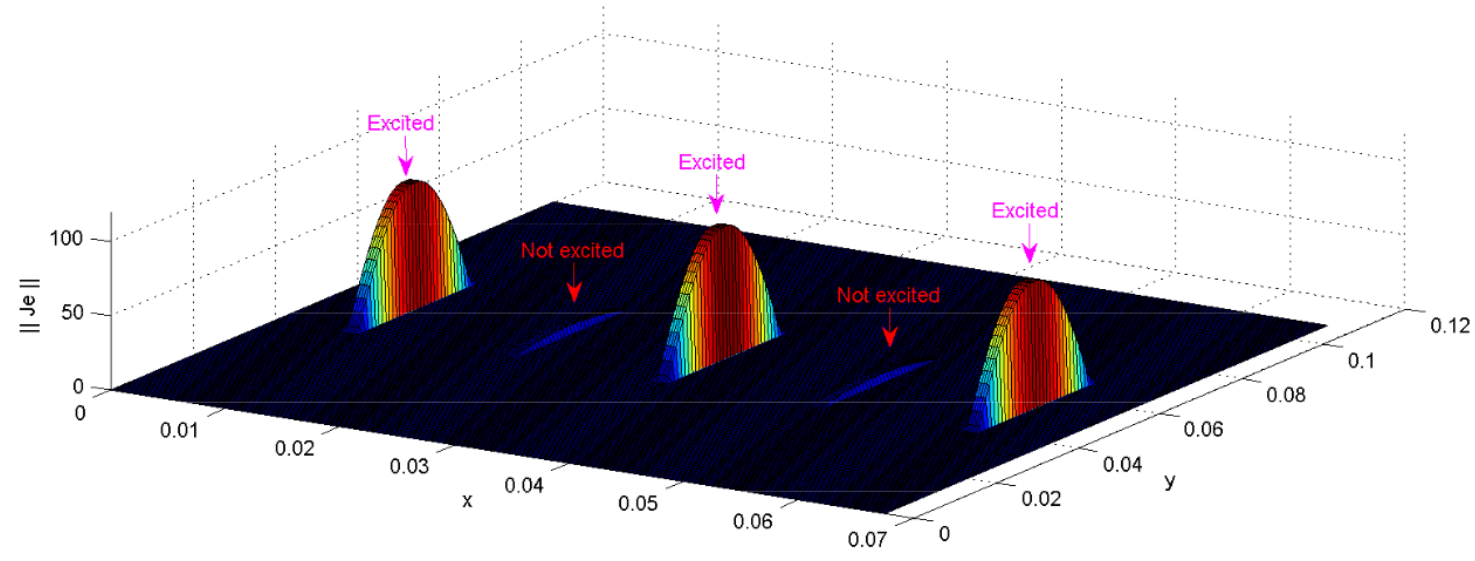

Figure 12. Current density of $1 \mathrm{D}$ aperiodic array's example (half wave dipoles )defined on the waveguide's aperture, described by the test functions at the operating frequency $\mathrm{f}=5.4 \mathrm{GHz}$. The used physical parameters are: $\alpha_{0}=0 \operatorname{rad} m^{-1}, \beta_{0}=0 \operatorname{rad~} m^{-1}, w=1 \mathrm{~mm}, \delta=0.75 \mathrm{~mm}\left(w \ll \lambda_{0}, \delta \ll\right.$ $\left.\lambda_{0}\right), d_{x}=13.5 \mathrm{~mm}, d_{y}=108 \mathrm{~mm}, L=\frac{\lambda_{0}}{2} \approx 27 \mathrm{~mm}, h=1.25 \mathrm{~mm}$ and $\epsilon=\epsilon_{r}=1$ (air).

A good comparison is achieved between both periodic and aperiodic structures in terms of the radiation pattern, as given in the figure(20). To validate this latter result an array factor formulation is proposed, which is applicable for the periodic array containing isotropic elements with separation $d_{x} \geq \frac{\lambda}{2}$.

The figure (21) shows the radiation beam steering (with $\phi_{s}=0^{\circ}, \theta_{s}=45^{\circ}$ ) of different periodic and aperiodic arrays, and explains how to reduce side lobes levels by varying the voltage configurations. As seen, the proposed antennas structures have a good beam steering property which is highly effective to cover the spherical beam coverage. 

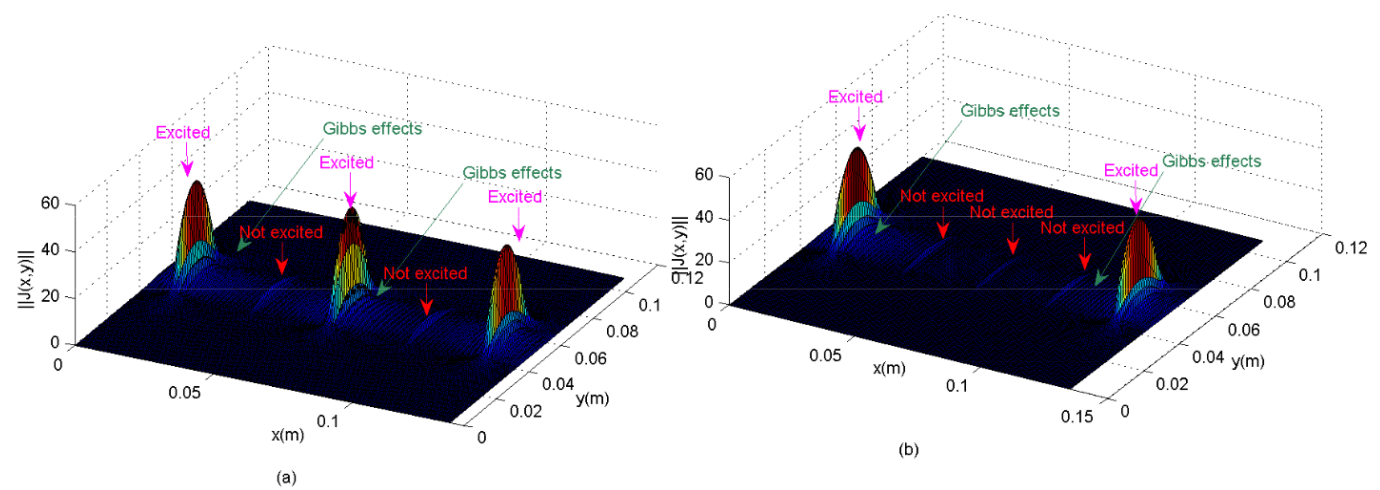

Figure 13. Current densities examples of $1 \mathrm{D}$ aperiodic arrays (half wave dipoles )that appeared on the guide wave's aperture and described by the basis functions (Guide's modes) (Without convergence) at the operating frequency $\mathrm{f}=5.4 \mathrm{GHz}$. The used physical parameters are: $\alpha_{0}=0 \mathrm{rad} m^{-1}, \beta_{0}=0 \mathrm{rad} m^{-1}$, $w=1 \mathrm{~mm}, \delta=0.75 \mathrm{~mm}\left(w \ll \lambda_{0}, \delta \ll \lambda_{0}\right), d_{x}=27 \mathrm{~mm}, d_{y}=108 \mathrm{~mm}, L=\frac{\lambda_{0}}{2} \approx 27 \mathrm{~mm}, h=1.25 \mathrm{~mm}$ and $\epsilon=\epsilon_{r}=1$ (air).

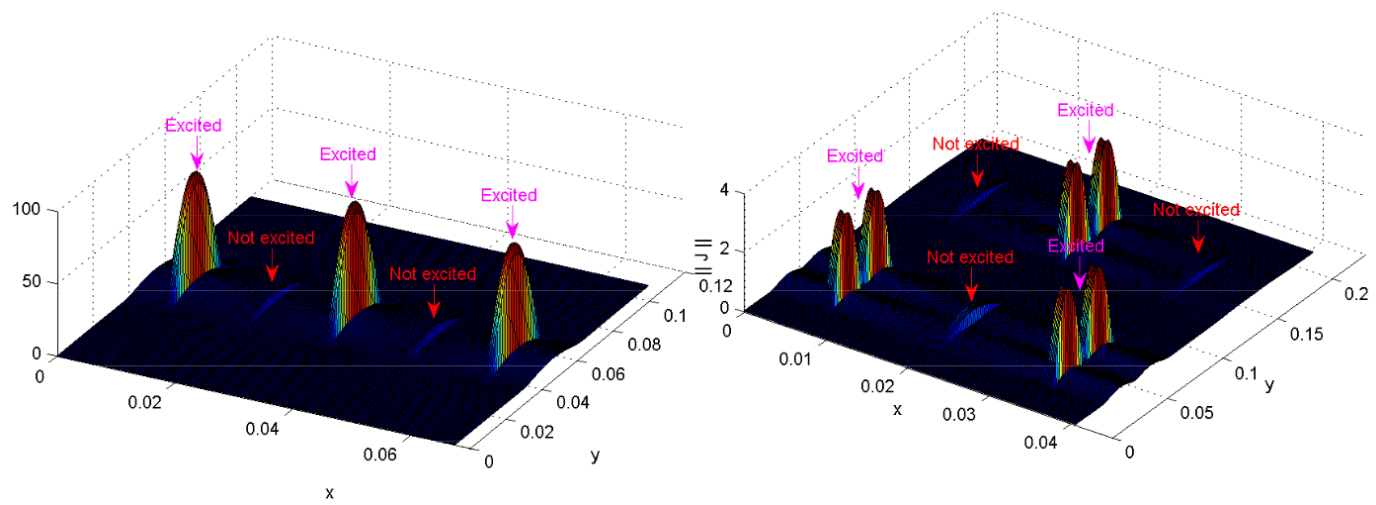

Figure 14. Current densities 1D and 2D of aperiodic arrays's examples (half wave dipoles) that lying on the guide wave's aperture and described by the basis functions (guide's modes) at the operating frequency $\mathrm{f}=5.4 \mathrm{GHz}$. The used physical parameters are: $\alpha_{0}=0 \mathrm{rad} m^{-1}, \beta_{0}=0 \mathrm{rad} m^{-1}, w=$ $1 \mathrm{~mm}, \delta=0.75 \mathrm{~mm}\left(w \ll \lambda_{0}, \delta \ll \lambda_{0}\right), d_{x}=13.5 \mathrm{~mm}, d_{y}=108 \mathrm{~mm}, L=\frac{\lambda_{0}}{2} \approx 27 \mathrm{~mm}, h=1.25 \mathrm{~mm}$ and $\epsilon=\epsilon_{r}=1$ (air).

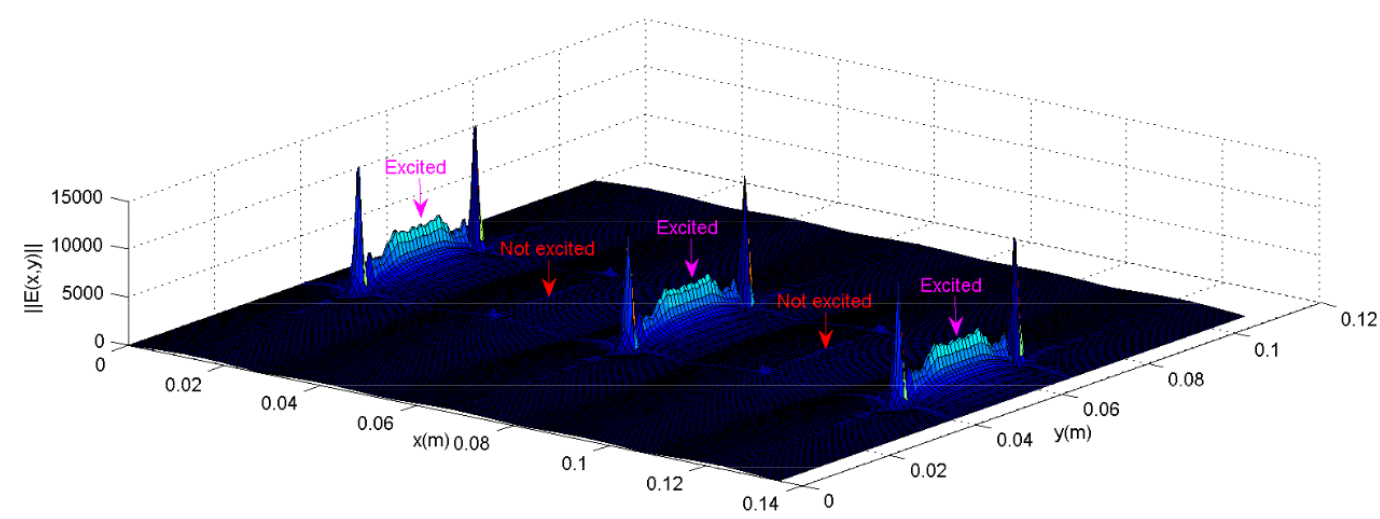

Figure 15. Distribution of the electric density for (5x1) aperiodic phased half-wavelength planar dipoles (with $(1,0,1,0,1)$ voltage configuration) described with the basis functions (guide's modes) at $\mathrm{f}=5.4 \mathrm{Ghz}$ (using EEEE electric walls). 


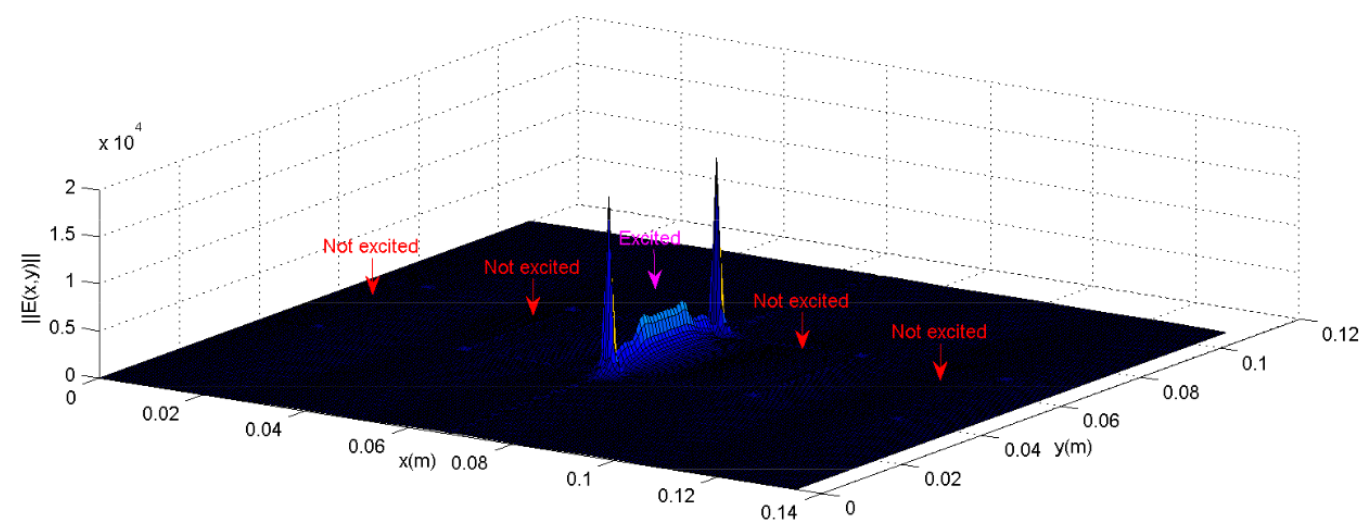

Figure 16. Distribution of the electric density for (5x1) aperiodic phased half-wavelength planar dipoles (with $(0,0,1,0,0)$ voltage configuration) described with the basis functions (guide's modes) at $\mathrm{f}=5.4 \mathrm{Ghz}$ (using EEEE electric walls).

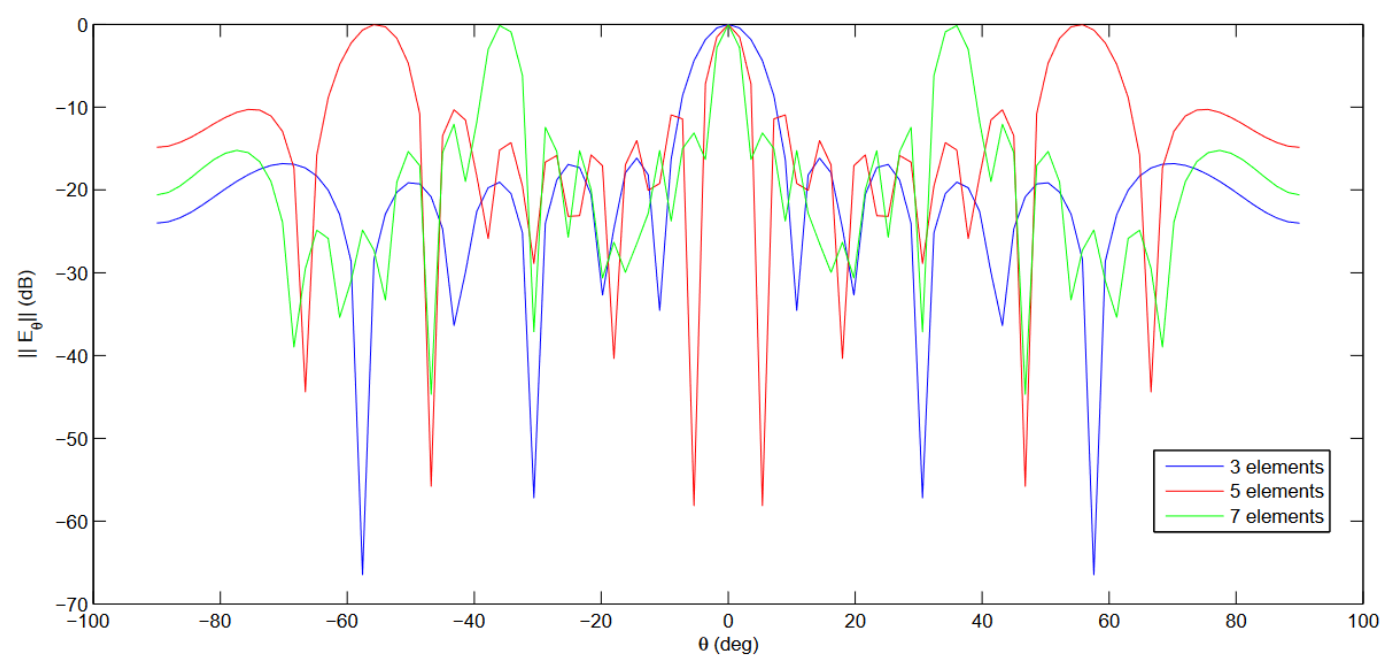

Figure 17. Radiation pattern computed with a MoM-GEC method against the motifs number at the operating frequency $\mathrm{f}=5.4 \mathrm{GHz}$ (Periodic array)

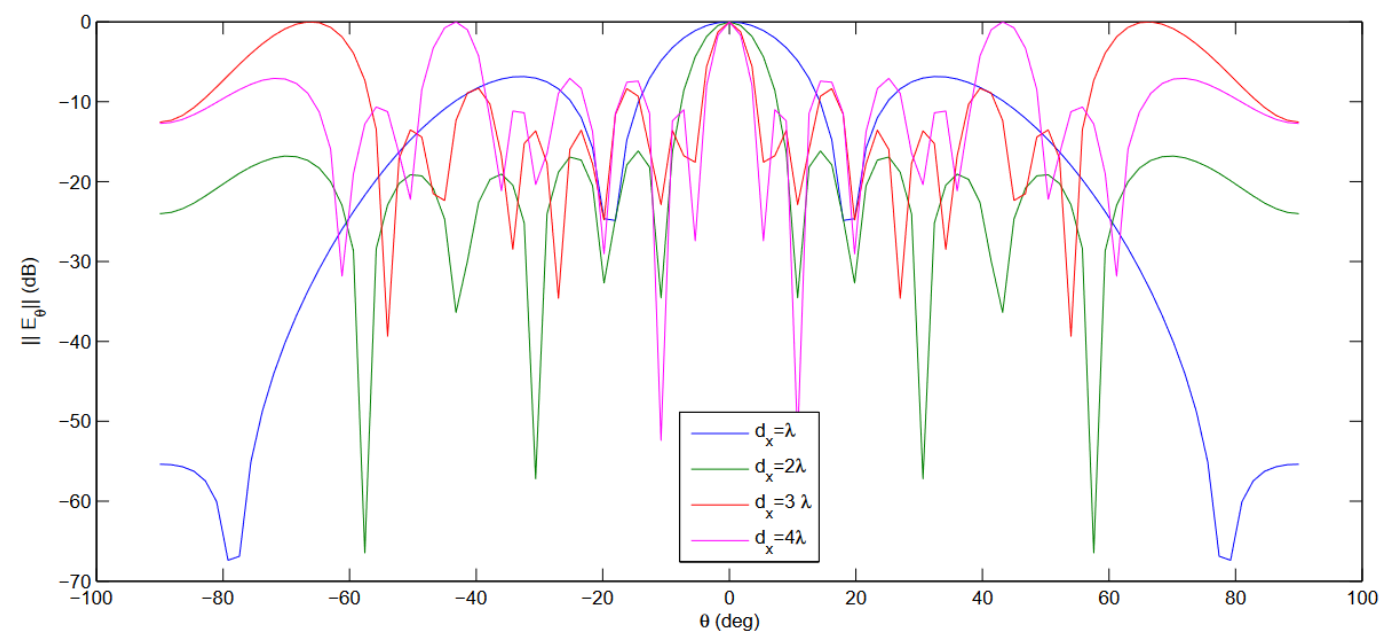

Figure 18. Radiation pattern computed with a MoM-GEC method against the periods at the operating frequency $\mathrm{f}=5.4 \mathrm{GHz}$ (Periodic array) 


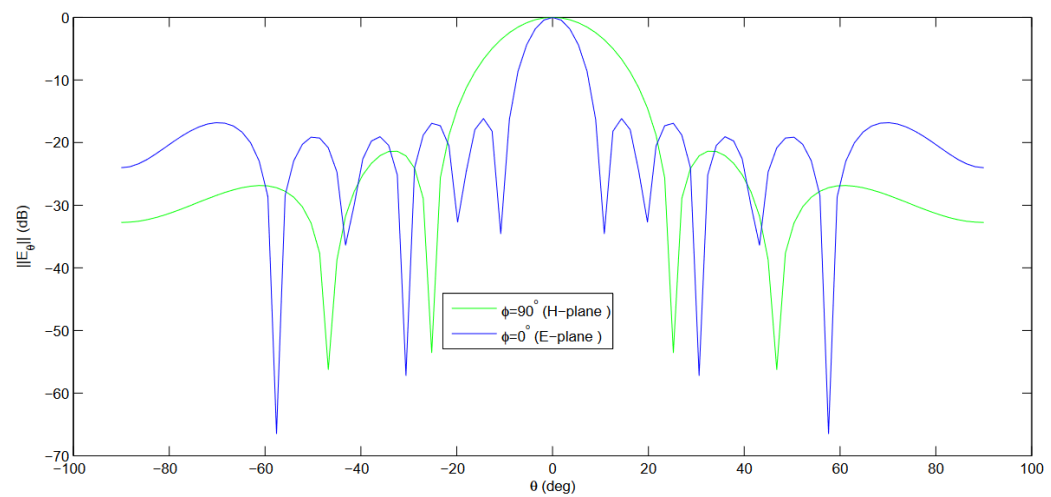

Figure 19. E-and H-plane cuts of the radiation pattern computed with a MoM-GEC method at the operating frequency $\mathrm{f}=5.4 \mathrm{GHz}$ (Periodic array)

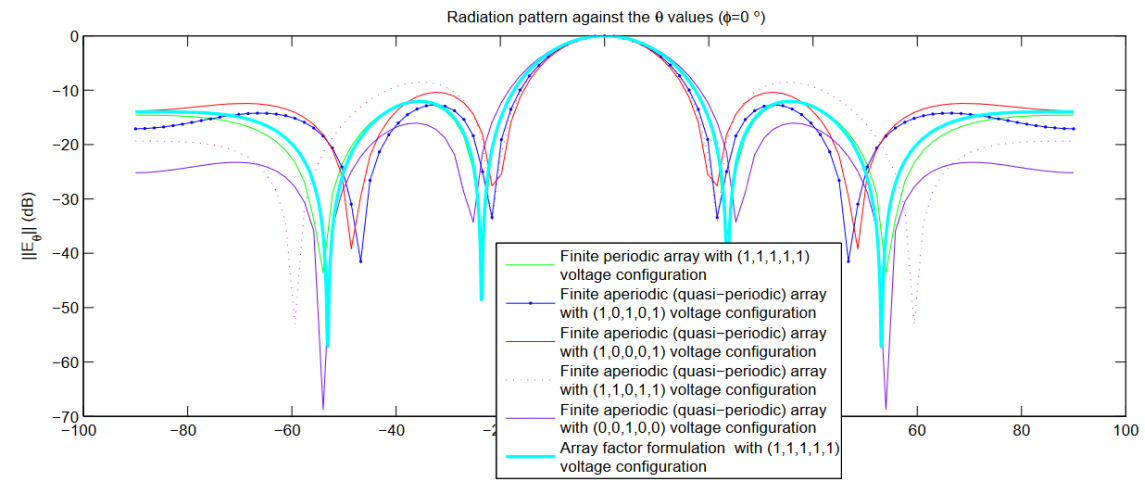

Figure 20. Radiation pattern for distinct aperiodic configurations compared to periodic arrays using electromagnetic and analytic formulations (MoM-GEC and analytic formulations )
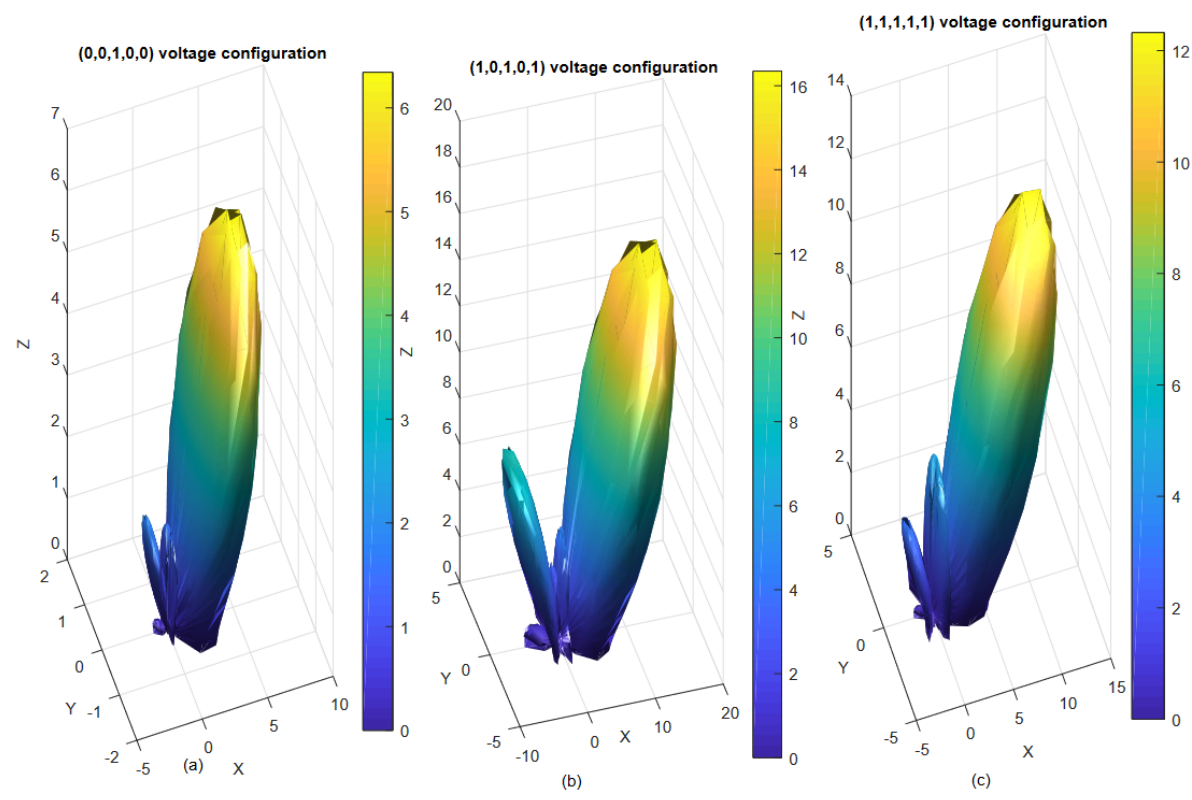

Figure 21. 3D Electronically scanned radiation pattern examples for distinct aperiodic array configurations compared to periodic array using the electromagnetic calculation (MoM-GEC method): $\phi_{s}=$ $0^{\circ}, \theta_{\mathcal{S}}=45^{\circ}$ angles of steering 


\subsection{Artificial Neural Network (ANN) Application}

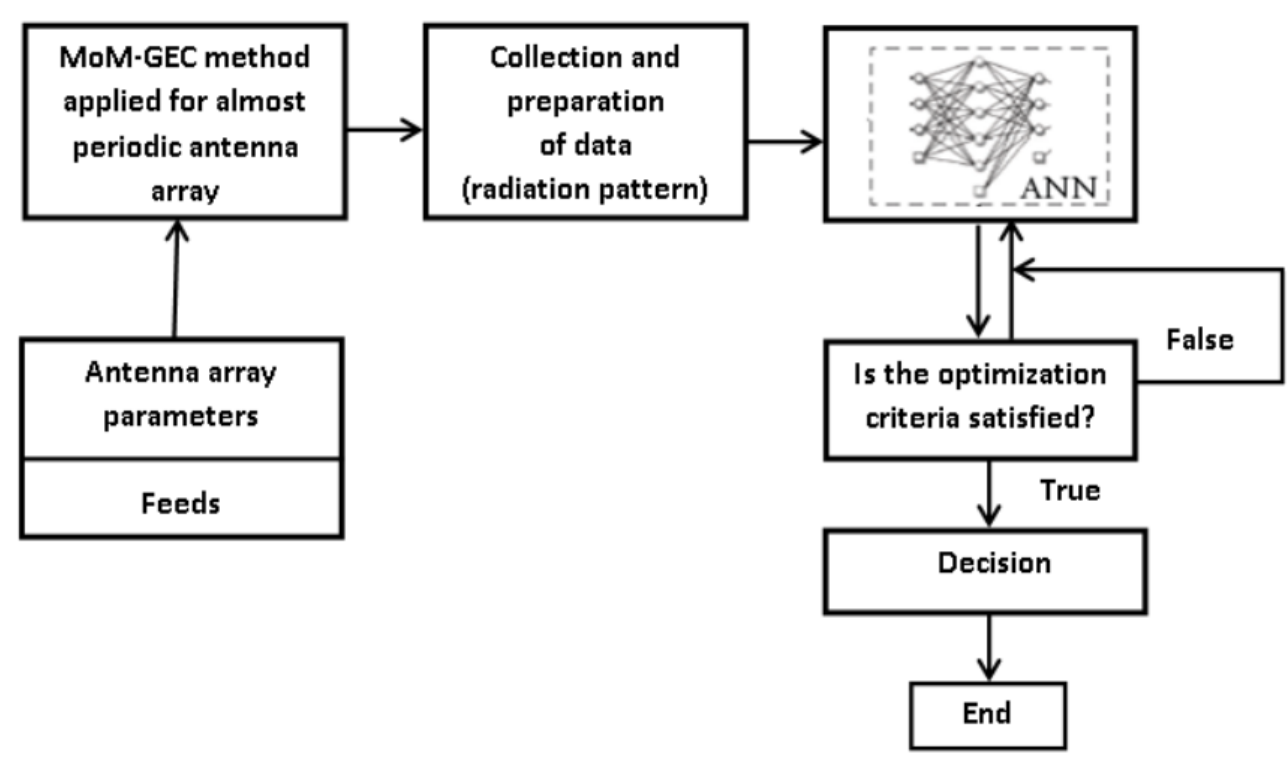

Figure 22. Flow diagram illustrating the numerical radiation pattern optimization using artificial neural network algorithm

The diagram presented in the figure(22) resumes the applied process to generate the optimized radiation pattern. Firstly, the necessary data collection is prepared for training employing a numerical calculation obtained by the moment method combined with an equivalent circuit, when the parameters of the almost periodic array structure are given. Then, the architectural parameters of the default ANN model are established and, a comprehensive analysis of the ANN training is carried out in order to develop a predictive performance. To propose an optimal ANN representation of high performance and to improve generalization capabilities of the ANN models, the early stopping (ES) method is mainly used in ANN training [21].

In general, two frequently used ways to overcome the overtraining condition, i.e., to decide when to terminate the training process, are the early stopping (ES) and regularization methods. Early stopping (ES) is typically used because it is easy to be understood and implemented and has been appreciated over the regularization methods. In order to utilize the ES method, the existing data must be divided into three sets [18]:

1. The training set was used to determine ANN weights.

2. The validation set was used to check the ANN performance and decide when to stop the training process.

3. The test set was used to assess the performance capabilities of the developed ANN model.

Next, a more detailed explanation of the ES method is illustrated graphically in the figures (23),(24)and (25). The methodology of the ES approach can be found in [18,21].

As seen in figures (24) and (25), a good accuracy improvement generalization of the normalized radiation field is proven with the Early Stopping (ES) technique. However, an artificial neural network (ANN) based on the ES method (Network with early stopping) is able to generate very fast the results of synthesis compared to default artificial neural network (ANN) (Network without early stopping) which requires much more CPU time and memory. In consequence, the network with the ES method can better adapt to the test data set with fewer divergences, so the early stopping feature can be used to prevent the over adaptation of the network towards the training data.

To obtain the computation time acceleration, the difference in consumption time between 
the default training and training with the early stopping method (using the Levenberg algorithm) is given in the table below (2). It shows that the time consuming of an early stopping method is less than the time required for a default training (without early stopping).

Table 2. Time consumption learning presented on the overall progress of the artificial neural network (ANN)

\begin{tabular}{cc}
\hline Training & time consumed by the algorithm (seconds) \\
\hline Default training (with levenberg algorithm) & 126.863 \\
\hline $\begin{array}{c}\text { Training with early stopping method (using } \\
\text { levenberg algorithm) }\end{array}$ & 19.276 \\
\hline
\end{tabular}

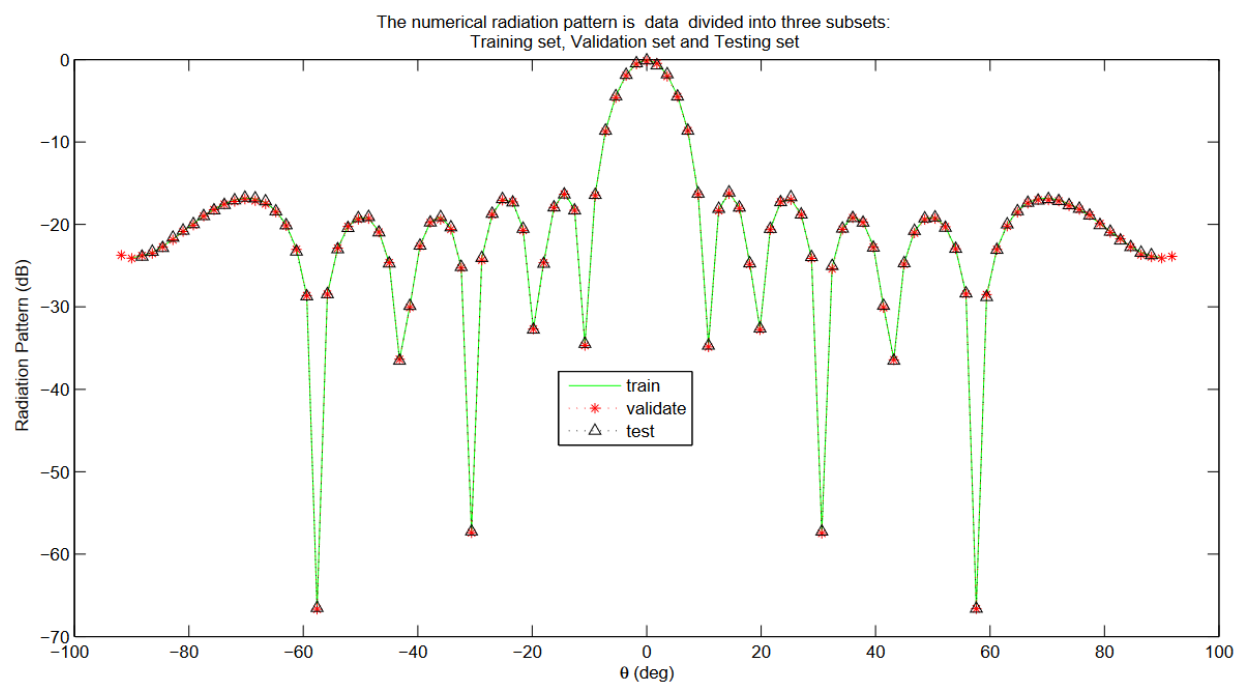

Figure 23. Radiation field is data divided into three subsets: Training set, Validation set and Testing set. The parameters which chosen to simulate the suggested almost periodic array antennas are $: \phi=0 \mathrm{deg}, N_{x}=3$ elements, and $d_{x}=2 \lambda=108 \mathrm{~mm}$

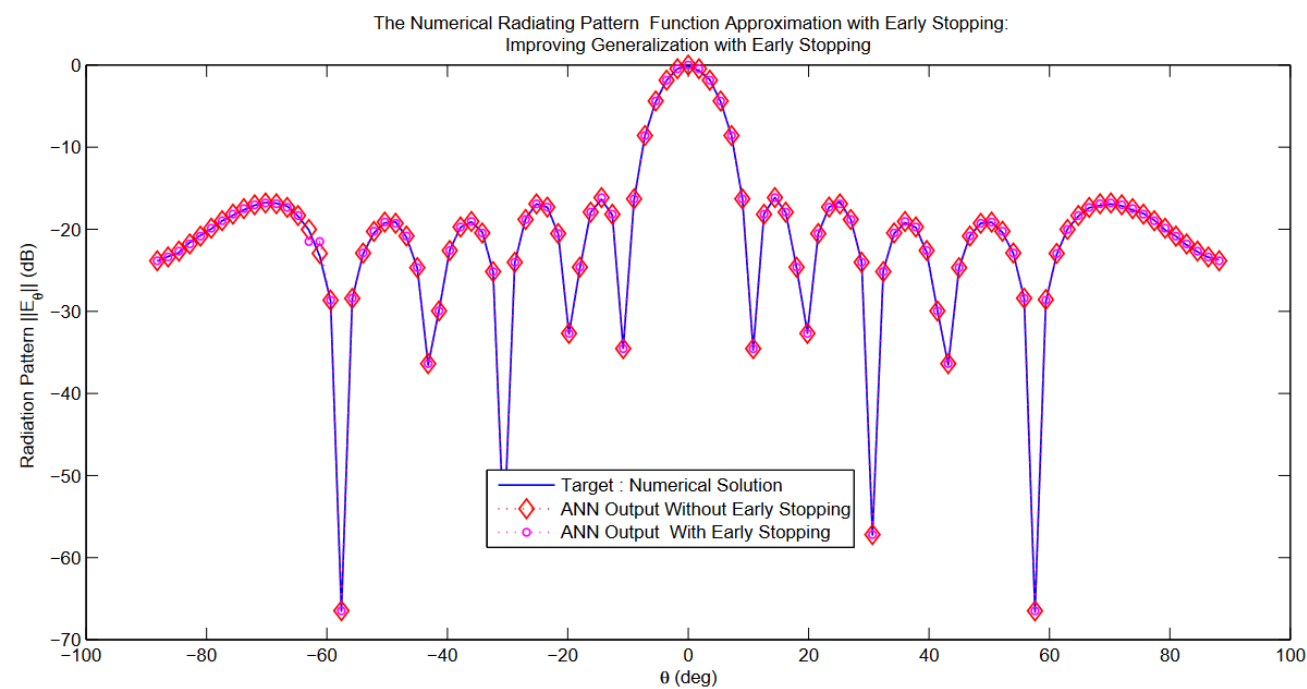

Figure 24. Radiation pattern function approximation with Early Stopping(ES): Improving Generalization with Early Stopping (ES).The parameters which chosen to simulate the suggested almost periodic array antennas are : $\phi=0 \mathrm{deg}, N_{x}=3$ elements, and $d_{x}=2 \lambda=108 \mathrm{~mm}$ (For periodic array example) 


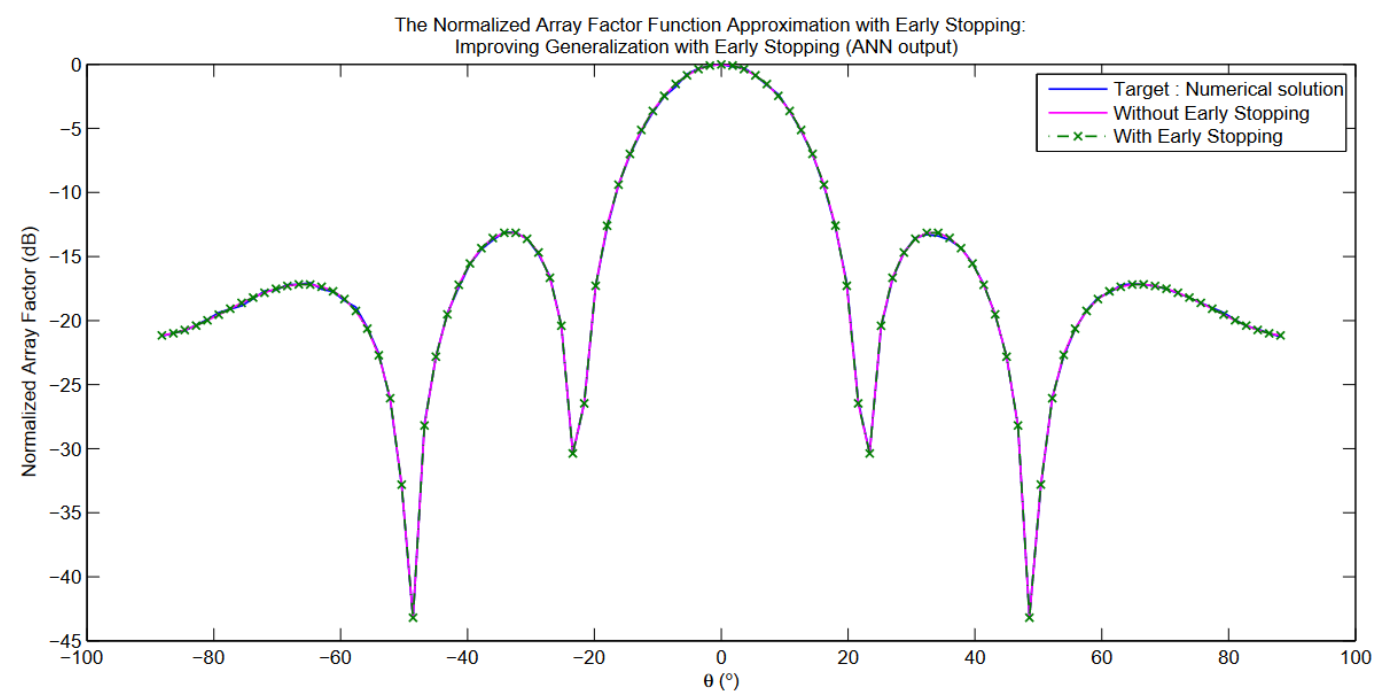

Figure 25. The ANN output as radiation pattern with Early Stopping(ES)( Improving Generalization with Early Stopping (ES)): For (5x1) aperiodic phased half-wavelength planar dipoles with (10101) voltage configuration

Table 3. Total CPU time (in seconds) used by electromagnetic calculation optimized through ANN algorithm

\begin{tabular}{ccc}
\hline $\begin{array}{c}\text { EM calculation using ANN } \\
\text { optimization }\end{array}$ & $\begin{array}{c}\text { ANN with spatial MoM } \\
\text { coding }\end{array}$ & $\begin{array}{c}\text { ANN with Floquet MoM } \\
\text { coding }\end{array}$ \\
\hline $\begin{array}{c}\text { Elapsed CPU Time (in } \\
\text { seconds) }\end{array}$ & 2704.502344 & 1750.368348 \\
\hline
\end{tabular}

Following the table (3), the optimization and synthesis using ANN with Floquet moment method $[9,11,14]$ is favored than the ANN with the spatial method, when the used motifs are considered coupled, because of the consumed CPU time of the modal analysis is more efficient and short compared to the old spatial MoM.

As the elapsed CPU time, the used memory is proven and discussed in our previous work, where the modal Floquet analysis remains more appreciated in comparison to the classical spatial MoM [9,11,14].

\subsection{Artificial Neural Network (ANN) Performance}

When the training process and the application of Multilayer Neural Network are complete, the network performance is checked and determines if any changes are needed to be implemented into the training algorithm, into the network architecture, or the data sets. It measures the network's performance according to the mean of squared errors. The accuracy of the artificial neural network (ANN) models is evaluated by the mean sum of squared error (MSE) between the(targets, outputs) values for the training data set. A plot of the training errors as shown in the following figure(26), respectively, the mean of squared error of the aperiodic configuration is presented in (27). This training is stopped when the training error increases against iterations to converge to the optimal solution. As clear, the training process for the artificial neural network (ANN) model is determined at 31 training epochs when MSE's best validation performance reaches the value of 0.12953 (Respectively, for the aperiodic structure, the MSE best validation performance value is 0.59252 about at 11 training epochs). The early stopping technique is finished if the validation parameter max fail reached a maximum 11 validation checks at epoch 40, with the gradient descent value 0.27225 (Whereas the gradient decent value of the aperiodic structure is 0.0475 when the max fail reached a maximum 11 validation checks at epoch 11), with reasonable $\mathrm{Mu}$ value 1.00 which would cause the convergence of the network fast, as presented in the 
overall progress of the artificial neural network (ANN)[18,24,25].

The next validation step in the network is to create a regression plot showing the relationship between the outputs of the network and the targets. If the training is perfect, the network outputs and the targets would be accurately equal, but the relationship is rarely perfect in practice. The dotted line in the training graph represents the perfect result: outputs $=$ targets. The solid line indicates the best-fit linear regression line between the outputs and the targets. The $\mathrm{R}$-value is an indication of the relationship between the outputs and the objectives. If $R=1$, this indicates that there is an exact linear relationship between outputs and targets. If $\mathrm{R}$ is close to zero, there is no linear relationship between outputs and targets. In this example, the training data indicates a good fit. The regression analysis was performed in an artificial neural network (ANN) model then the network concludes training. It is a statistical process for estimating the relationships between the output and target of the network. The plot regression function takes two parameters (targets, outputs) values and plots the linear. From all the presented graphs of the figure (28)(with the same manner for the aperiodic antenna array), the best regression value for validating the data is equal to $\mathrm{R}=0.99993$ (Concerning the aperiodic array $\mathrm{R}=0.9958$ ), regression value for testing the data is defined as $\mathrm{R}=0.99996$ (As for the aperiodic array $\mathrm{R}=0.99993$ ), regression value for training the data is defined as $\mathrm{R}=1$ and overall regression value is $\mathrm{R}=0.99996$ (as regards the aperiodic array $\mathrm{R}=0.99857$ ). This proves the developed model and the network procedure of training, testing and validation are significantly valid [26].

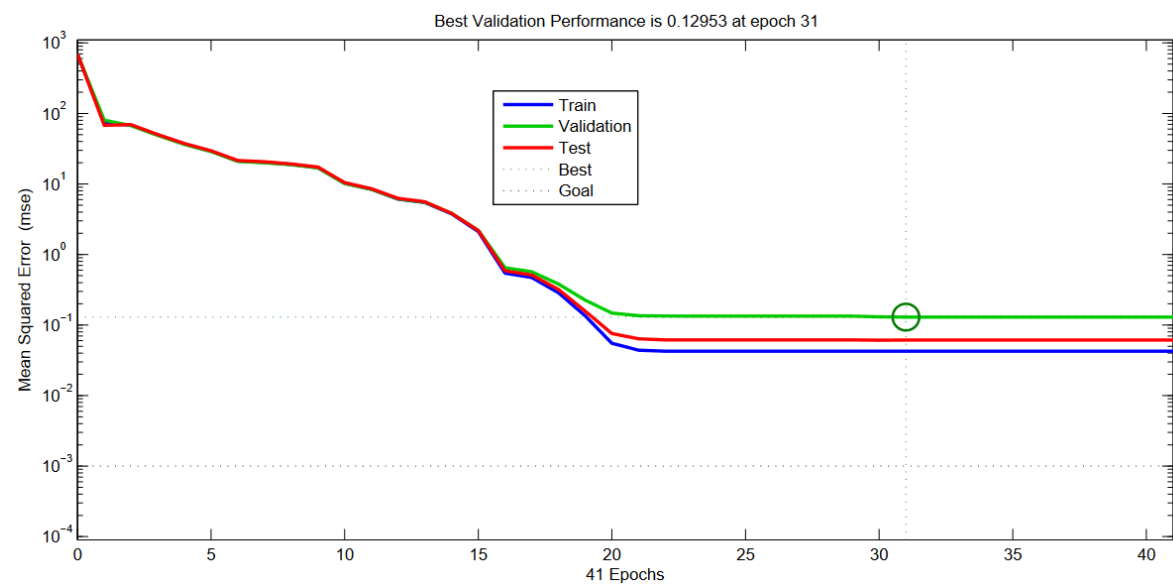

Figure 26. Evaluation of mean squared error (For periodic array example)

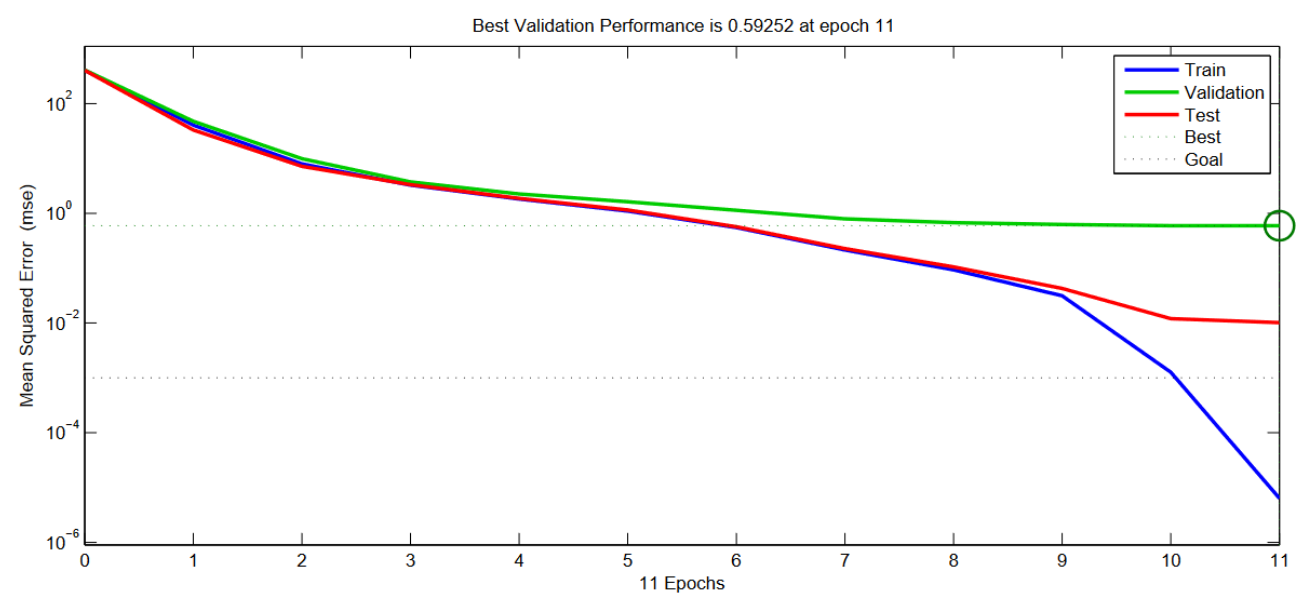

Figure 27. Evaluation of mean squared error (For quasi-periodic array example) 


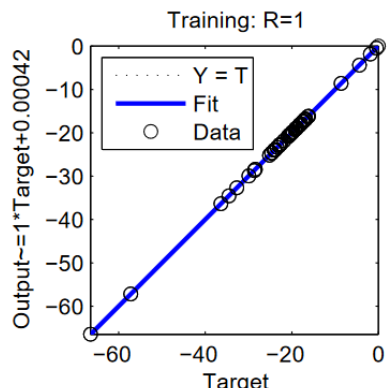

Test: $\mathrm{R}=0.99996$

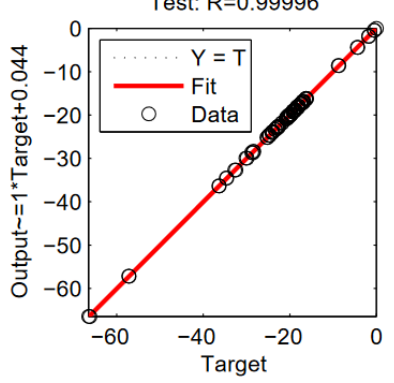

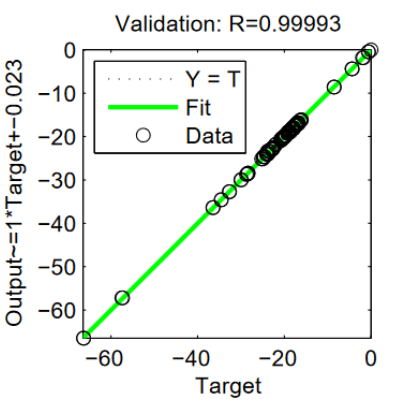

All: $\mathrm{R}=0.99996$

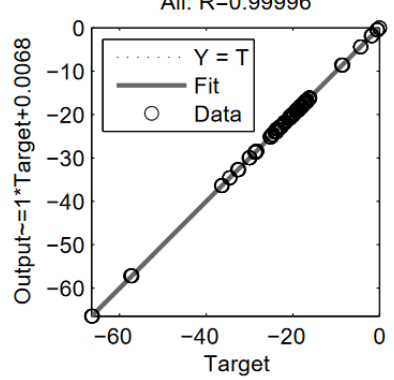

Figure 28. Regression plot of network (For periodic array example)

\section{Conclusion}

This work is interested to synthesize the radiation pattern of the planar almost periodic arrays including mutual couplings effects that developed and modeled by the known moment method combined with Generalized Equivalent Circuit (MoM-GEC). This investigation uses a novel algorithm based on neural technique as a computational model to occur the array pattern.

Many advantages are shown for synthesizing the calculated numerical radiation pattern using Artificial Neural Network algorithm, for example, :

- Reducing the computing time and storage memory, especially by adopting the early stopping method that eliminates the overfitting problem.

- Suitable to be used for a coupled and complex quasi-periodic configuration.

- Simple and easy for implementation than others optimization techniques (genetic, LMS,...etc).

- Adaptable to complex electromagnetic calculations taking into account the effects of mutual coupling.

This analysis is the starting basis for future work on neural network solutions for the synthesis of non-uniform, irregular, and sparse antenna arrays.

For future research, other optimization techniques (genetic, LMS,...etc) are suggested to be applied in complex almost periodic arrays to predict the desired radiation that can be adopted to increase the gain and to scan the range by suppressing the sidelobe level (SLL).

Author Contributions: Conceptualization, H.B; methodology, H.B. and A.T.; software, H.B. and A.T.; validation, H.B. and A.T.; formal analysis, H.B.; investigation, H.B. and A.T.; resources, A.T.; data curation, H.B. and A.T.; writing-original draft preparation, H.B. and A.T.; writing-review and editing, H.B. and A.T.; visualization, H.B. and A.T.; supervision, A.T.; project administration, A.T.; funding acquisition, A.T. All authors have read and agreed to the published version of the manuscript

Funding: This project received a part of the funding from the Laboratory Sys'Com-ENIT (LR-99ES21)-National Engineering School of Tunis ENIT, Tunis, Tunisia, 1002.

Data Availability Statement: Not applicable.

Conflicts of Interest: The authors declare no conflict of interest.

\section{Abbreviations}




$\begin{array}{ll}\text { MoM-GEC } & \text { Method of Moment with Generalized Equivalent Circuits } \\ \text { FSS } & \text { Frequency Selective Surfaces } \\ \text { EEEE } & \text { Electric walls } \\ \text { EMEM } & \text { Electric and Magnetic walls } \\ \text { EPEP } & \text { Electric and Periodic walls } \\ \text { PPPP } & \text { Periodic walls } \\ \text { 1-D } & \text { Uni-dimensional } \\ \text { 2-D } & \text { Two-dimensional } \\ \text { ANN } & \text { Artificial Neural Network } \\ \text { GA } & \text { Genetic Algorithm } \\ \text { ES } & \text { Early Stopping technique } \\ \text { MLP } & \text { Multilayer Perceptron } \\ \text { LMA } & \text { levenberg-Marquart Algorithm } \\ \text { MSE } & \text { Mean Squared Error } \\ \text { CPU } & \text { Central Processing Unit } \\ \text { LMS } & \text { Least Mean Squares algorithms }\end{array}$

\section{Appendix A}

\section{Midpoint Sums Approximating Double Integrals to calculate the far field [30]}

In this work, the proposed double integrals of the far-field expressions are evaluated by means of the midpoints sums approximation:

When

$$
S_{m n}=\sum_{i=1}^{n} \sum_{j=1}^{m} f\left(u_{i}, v_{j}\right) \triangle x \triangle y \approx \iint_{R} f(x, y) d x d y
$$

With

$$
u_{i}=a+\frac{i-1}{2} \triangle x
$$

And

$$
v_{j}=b+\frac{j-1}{2} \triangle y
$$

$u_{i}$ and $v_{j}$ are the midpoints of the ij-aperture subrectangle $\left[x_{i-1}, x_{i}\right]\left[y_{j-1} y_{j}\right]$.

Denote the midpoints of the ith x-subinterval and the jth $y$-subinterval (respectively), then $\triangle x$ and $\triangle y$ are the $\mathrm{m}$ and $\mathrm{n}$ subintervals length of the waveguide aperture.

\section{Appendix B}

\section{Matlab's code to express the far-field (radiation pattern) cuts [29]}

Here, the main Matlab code used to calculate the far-field expression (based on the Fourier transform) that obtained by the moment method simplified by the equivalent circuit (electromagnetic calculation): Matlab's code to express the far-field (radiation pattern) cuts

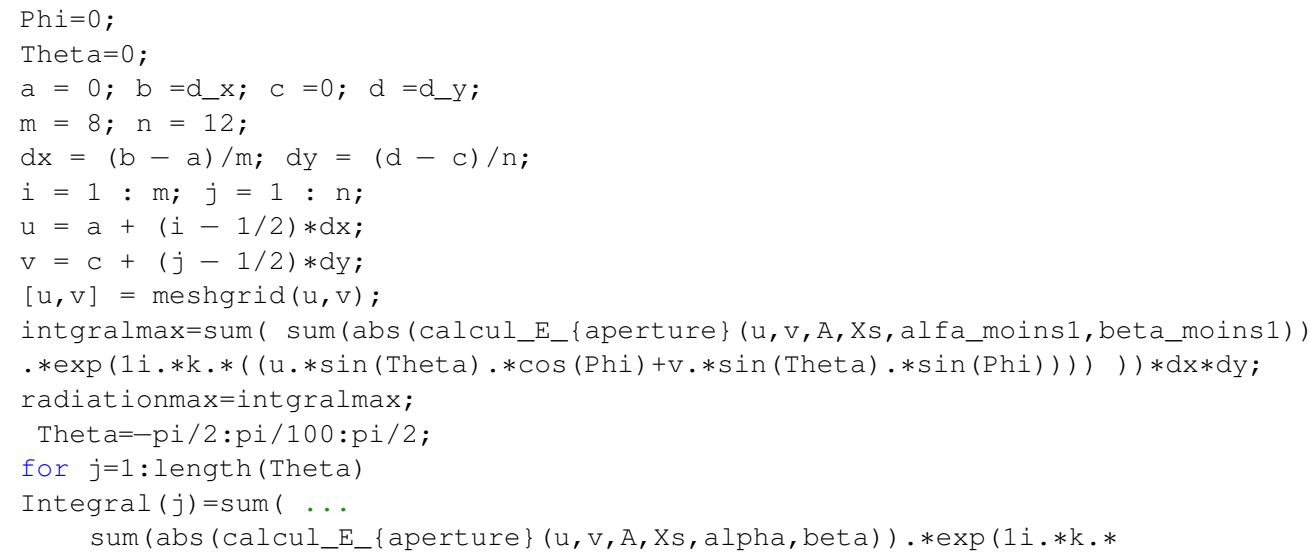




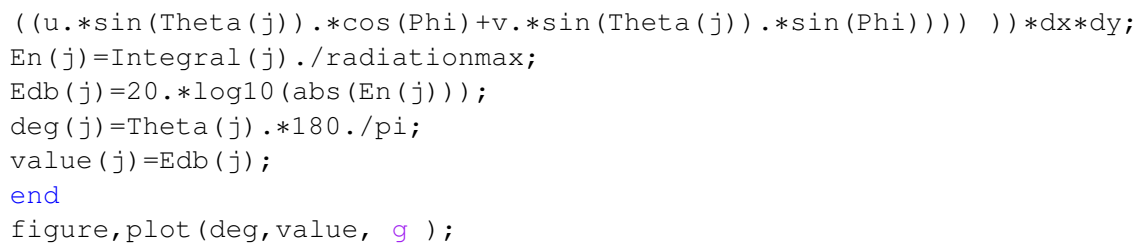

Where Calcul E aperture $(\mathrm{u}, \mathrm{v}, \mathrm{A}, \mathrm{Xs}$, alpha,beta) is the radiating field calculated at the waveguide's aperture (discontinuity plane) through the moment method combined by the equivalent circuit (as described in the figures 11,15 and 16).

\section{References}

1. Hamdi, B.; Nouainia, A.; Aguili, T.; Baudrand, H. ANN Synthesis and Optimization of Electronically Scanned Coupled Planar Periodic and Aperiodic Antenna Arrays Modeled by the MoM-GEC Approach. In 2020 IEEE Eighth International Conference on Communications and Electronics (ICCE) - MICROWAVE ENGINEERING, PHU QUOC ISLAND, VIETNAM; Jan 13, 2021 - Jan 15, 2021. Available online: https://vixra.org/pdf/2111.0161v1.pdf

2. Sagar, M.S.I.; Ouassal, H.; Omi, A.I.; Wisniewska, A.; Jalajamony, H.M.; Fernandez, R.E.; Sekhar, P.K. Application of Machine Learning in Electromagnetics: Mini-Review. Electronics 2021, 10, 2752. https:/ / doi.org/10.3390/electronics10222752

3. Taghvaee, H., 2021. On scalable, reconfigurable, and intelligent metasurfaces.

4. Singh, A., ; Singh, S. Design and optimization of a modified Sierpinski fractal antenna for broadband applications. Applied Soft Computing 2016, 38, 843-850.

5. Hamdi, B.; Aguili, T.; Baudrand, H. Uni-dimensional planar almost periodic structures analysis to decompose central arbitrary located source in spectral domain. In 201215 International Symposium on Antenna Technology and Applied Electromagnetics (ANTEM, Toulouse (France) ,2012, June) (pp. 1-5). IEEE.

6. Mekkioui, Z.; Baudrand, H. A full-wave analysis of uniform microstrip leaky-wave antenna with arbitrary metallic strips. Electromagnetics 2008, 28(4), 296-314.

7. Mekkioui, Z.; Baudrand, H. Effects of multilayers superstrates on microstrip leaky-wave antennas radiating characteristics and performances. Electromagnetics 2005, 25(2), 133-151.

8. Mekkioui, Z.; Baudrand, H. Analyse rigoureuse d'antenne diélectrique microruban uniforme à ondes de fuite. In Annales des télécommunications, vol. 57, no. 5, pp. 540-560. Springer-Verlag, 2002.

9. Hamdi, B.; Aguili, T.; Raveu, N.; Baudrand, H. Calculation of the mutual coupling parameters and their effects in 1-D planar almost periodic structures. Progress In Electromagnetics Research B 2014, 59, 269-289.

10. Aidi, M.; Hajji, M.; Ben Ammar, A.; Aguili, T. Graphene nanoribbon antenna modeling based on MoM-GEC method for electromagnetic nanocommunications in the terahertz range. Journal of ElEctromagnEtic WavEs and applications 2016, 30(8), 1032-1048.

11. Hamdi, B.;Aguili, T.; Baudrand, H. Floquet modal analysis to modelize and study 2-D planar almost periodic structures in finite and infinite extent with coupled motifs. Progress In Electromagnetics Research B 2015, 62, 63-86.

12. Bilel, H.; Taoufik, A. Floquet Spectral Almost-Periodic Modulation of Massive Finite and Infinite Strongly Coupled Arrays: Dense-Massive-MIMO, Intelligent-Surfaces, 5G, and 6G Applications. Electronics 2022, 11(1), 36.

13. Bilel, H.; Selma, L.; Taoufik, A. Artificial neural network (ANN) approach for synthesis and optimization of (3D) three-dimensional periodic phased array antenna. In 2016 17th International Symposium on Antenna Technology and Applied Electromagnetics (ANTEM-Montreal (Canada) ),2016, July, (pp. 1-6). IEEE.

14. Mekkioui, Z.; Baudrand, H. Bi-dimensional bi-periodic centred-fed microstrip leaky-wave antenna analysis by a source modal decomposition in spectral domain. IET microwaves, antennas and propagation 2009, 3(7), 1141-1149.

15. Yan, K. K.; Lu, Y. Sidelobe reduction in array-pattern synthesis using genetic algorithm. IEEE Transactions on Antennas and Propagation 1997, 45(7), 1117-1122.

16. Dudczyk, J.; Kawalec, A. Adaptive forming of the beam pattern of microstrip antenna with the use of an artificial neural network. International Journal of Antennas and Propagation 2012, 2012.

17. Freni, A.; Mussetta, M.; Pirinoli, P. Neural network characterization of reflectarray antennas. International Journal of Antennas and Propagation, 2012.

18. Hamdi, B.; Limam, S.; Aguili, T. Uniform and concentric circular antenna arrays synthesis for smart antenna systems using artificial neural network algorithm. Progress In Electromagnetics Research B 2016, 67, 91-105.

19. Thevenot, M.; Menudier, C.; El Sayed Ahmad, A.; Zakka El Nashef, G.; Fezai, F.;Abdallah, Y.; Monediere, T. (2012). Synthesis of antenna arrays and parasitic antenna arrays with mutual couplings. International Journal of Antennas and Propagation, 2012.

20. Ayari, M.; Aguili, T.; Temimi, H.; Baudrand, H. An extended version of Transverse Wave Approach (TWA) for full-wave investigation of planar structures. Journal of Microwaves, Optoelectronics and Electromagnetic Applications (JMOe) 2008, 7(2), 123-138. 
21. Wang, L.; Quek, H. C.; Tee, K. H.; Zhou, N.; Wan, C. (2005, October). Optimal size of a feedforward neural network: How much does it matter?. In Joint International Conference on Autonomic and Autonomous Systems and International Conference on Networking and Services-(icas-isns' 05) (pp. 69-69). IEEE.

22. Kapetanakis, T. N.; Vardiambasis, I. O.; Liodakis, G. S.; Ioannidou, M. P.; Maras, A. M. Smart antenna design using neural networks. In 8th International Conference: New Horizons in Industry, Business and Education (NHIBE 2013, August) (pp. 130-135

23. Rawat, A.; Yadav, R. N.; Shrivastava, S. C. Neural network applications in smart antenna arrays: A review. AEU-International Journal of Electronics and Communications 2012, 66(11), 903-912.

24. Merad, L.; Bendimerad, F. T.; Meriah, S. M.; Djennas, S. A. Neural networks for synthesis and optimization of antenna arrays. RADIOENGINEERING-PRAGUE-2007, 16(1), 23.

25. Ghayoula, R.; Fadlallah, N.; Gharsallah, A.; Rammal, M. Phase-only adaptive nulling with neural networks for antenna array synthesis. IET microwaves, antennas propagation 2009, 3(1), 154-163.

26. Ali, B. A.; Salit, M. S.; Zainudin, E. S.; Othman, M. Integration of artificial neural network and expert system for material classification of natural fibre reinforced polymer composites. American Journal of Applied Sciences 2015, $12(3), 174$.

27. Ghayoula, E.; Ghayoula, R.; Haj-Taieb, M.; Chouinard, J. Y.; Bouallegue, A. Pattern Synthesis Using Hybrid Fourier-Neural Networks for IEEE 802.11 MIMO Application. Progress In Electromagnetics Research B 2016, 67, 45-58.

28. Vardaxoglou, J.C. Frequency Selective Surfaces, Analysis and Design; John Wiley and Sons: Hoboken, NJ, USA, 1997.

29. Das, Sisir K. Antenna and wave propagation. Tata McGraw-Hill Education, 2013.

30. 14.1 Project :Midpoint Sums Approximating Double Integrals (Using MATLAB) . Available online: https://people.math.aau.dk/ olav/undervisning/ide10/proj14-1.pdf

31. Costa, B.F. ; Abrao, T.,. Closed-form directivity expression for arbitrary volumetric antenna arrays. IEEE Transactions on Antennas and Propagation 2018, 66(12), pp.7443-7448.

32. Valerio, G.; Baccarelli, P.; Burghignoli, P.; Galli, A.; Rodríguez-Berral, R.; Mesa, F. Analysis of periodic shielded microstrip lines excited by nonperiodic sources through the array scanning method. Radio Science 2008, 43(01), pp.1-15. 